\title{
Exemplaric Expressivity of Modal Logics ${ }^{\star}$
}

\author{
Bart Jacobs $^{1}$ and Ana Sokolova ${ }^{2 \star \star}$ \\ ${ }^{1}$ Institute for Computing and Information Sciences, Radboud University Nijmegen \\ P.O. Box 9010, 6500 GL Nijmegen, The Netherlands. \\ Email: B. Jacobsecs.ru.nl \\ URL: http://www.cs.ru.nl/B.Jacobs \\ 2 Department of Computer Sciences, University of Salzburg \\ Jakob-Haringer-Str. 2, 5020 Salzburg, Austria. \\ Email: Ana.Sokolova@cs.uni-salzburg.at \\ URL: http://www.cs.uni-salzburg.at/ anas
}

May 23, 2008

\begin{abstract}
This paper investigates expressivity of modal logics for transition systems, multitransition systems, Markov chains, and Markov processes, as coalgebras of the powerset, finitely supported multiset, finitely supported distribution, and measure functor, respectively. Expressivity means that logically indistinguishable states, satisfying the same formulas, are behaviourally indistinguishable. The investigation is based on the framework of dual adjunctions between spaces and logics and focuses on a crucial injectivity property. The approach is generic both in the choice of systems and modalities, and in the choice of a "base logic". Most of these expressivity results are already known, but the applicability of the uniform setting of dual adjunctions to these particular examples is what constitutes the contribution of the paper.
\end{abstract}

\section{Introduction}

During the last decade, coalgebra $[30,17]$ has become accepted as an abstract framework for describing state-based dynamic systems. Fairly quickly it was recognised, first in [26], that modal logic is the natural logic for coalgebras and also that coalgebras provide obvious models for modal logics. Intuitively there is indeed a connection, because modal operators can be interpreted in terms of next or previous states, with respect to some transition system or, more abstractly, coalgebra. The last few years have shown a rapid development in this (combined) area [23,16,27,22,24,5,31,32,29,7,19]. One of the more interesting aspects is the use of dualities or dual adjunctions.

Here is a brief "historical" account of how the emergence of dual adjunctions in logical settings can be understood. For reasoning about functors the idea of predicate lifting was used already early in $[14,15]$. This involves the extension of a predicate (formula) $P \subseteq X$ to a lifted predicate $\bar{P} \subseteq T X$, for an endofunctor $T$ : Sets $\rightarrow$ Sets whose coalgebras $X \rightarrow T X$ we wish to study. The notion of invariant arises via such predicate

* To appear in: Journal of Logic and Computation.

${ }^{\star \star}$ Supported by the Austrian Science Fund (FWF) project P18913. 
liftings. Liftings can be described as a function $\mathcal{P} X \rightarrow \mathcal{P} T X$, or actually as a natural transformation $\mathcal{P} \Rightarrow \mathcal{P} T$, for the contravariant powerset functor $\mathcal{P}$ : Sets ${ }^{\text {op }} \rightarrow$ Sets, $c f$. [27]. With the introduction of polyadic modal operators [31,19] this natural transformation morphed into maps of the form $\coprod_{n \in \mathbb{N}}(\mathcal{P} X)^{n} \rightarrow \mathcal{P} T X$, or more abstractly via a functor $L$ into a natural transformation $L \mathcal{P} \Rightarrow \mathcal{P} T$. All this takes place in a situation:

$$
T \bigcirc \text { Sets }^{\text {op }} \underset{\mathcal{P}}{\rightleftarrows} \text { Sets } \bigcirc L \quad \text { with } L \mathcal{P} \stackrel{\sigma}{\Longrightarrow} \mathcal{P} T
$$

where we have a dual adjunction $\mathcal{P} \dashv \mathcal{P}$. The category Sets on the left describes the spaces on which we have coalgebra structures (of the functor $T$ ). Sets on the right describes the logical universe, on which there is a functor $L$ for modal operators.

The above dual adjunction thus provides the raw setting for considering coalgebras and their (modal) logics. For specific kinds of coalgebras (given by particular functors $T$ ) there may be more structure around. In this paper we shall study examples with the categories Sets of sets and Meas of measure spaces on the left in (1), and the categories BA of Boolean algebras and MSL of meet semilattices on the right. The latter capture Boolean logic and logic with only finite conjunctions, respectively. Section 2 will describe the adjunctions involved. Similar adjunctions have been used in process semantics (see e.g. [1]) or more generally in [18].

Section 3 will enrich these dual adjunctions with endofunctors like $T$ and $L$ in the above diagram (1). It also contains two "folklore" results about the natural transformation involved (the $\sigma$ in (1)). The most important one is Theorem 4 that relates a certain injectivity condition to the fundamental property of expressivity of the logic-which means that logically indistinguishable states are also behaviourally indistinguishable. This theorem is known for some time already in the community and has appeared in print in various places $[19,20,7]$, in one form or another. We present a convenient formulation (and proof) that is useful in our setting, but we do not claim it as our contribution.

What we do claim as contribution appears in Section 4. There we use Theorem 4 to prove expressivity for four concrete examples. In all these cases we describe appropriate modalities, and prove expressivity by adding:

- Boolean logic for image-finite transition systems, as coalgebras of the finite powerset functor on Sets;

- finite conjunctions logic for multitransition systems, as coalgebras of the finitely supported multiset functor on Sets;

- finite conjunctions logic for Markov chains, as coalgebras of the finitely supported discrete (sub)distribution functor on Sets;

- finite conjunctions for Markov processes, as coalgebras of the Giry functor on the category of measure spaces.

The first point goes back to [13]. Here we cast it in the framework of dual adjunctions, with an explicit description of the "modality" endofunctor $L$ on the category BA of Boolean algebras and its relevant properties. An expressivity result of graded modal logic (based on Boolean logic) for multitransition systems already exists [31]. 
There is also already an expressivity result for Markov chains with the standard modalities and Boolean logic (including negation), $c f$. [7,25]. Here, we give a proof that finite conjunctions suffice for expressivity for both multitransition systems and Markov chains, just as they do for non-discrete probabilistic systems [10,8]. Then we reformulate the expressivity result of $[10,8]$ within our uniform setting of dual adjunctions. Additionally we elaborate on the relation between the discrete and non-discrete Markov chains/processes and show precisely how expressivity for Markov processes yields expressivity for Markov chains, resulting in alternative indirect proofs for the third point.

Finally, we should emphasise that we include neither atomic propositions nor action labels in the logics and the systems, since we are interested in the essence of the expressivity results. This way we wish to prepare the ground for arbitrary (possibly modular) extensions in the setting of dual adjunctions.

\section{Dual adjunctions}

In this section we shall be interested in an adjoint situation of the form:

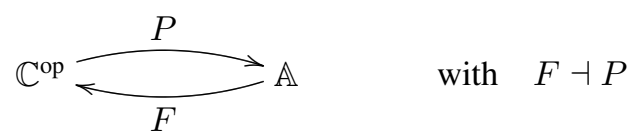

We informally call this a dual adjunction because one of the categories involved occurs naturally in opposite form. In the next section we shall extend such situations with endofunctors, on $\mathbb{C}$ for systems as coalgebras and on $\mathbb{A}$ for logics, but at this preparatory stage we only look at the adjunctions themselves.

Such situations (2) are familiar in duality-like settings, for instance with $\mathbb{C}=\mathbb{A}=$ Sets, and $P=F=$ "contravariant powerset", like in (1); with $\mathbb{C}=\operatorname{Sets}, \mathbb{A}=$ PreOrd, $P=$ "contravariant powerset", $F=$ "upsets"; or with $\mathbb{C}=$ "topological spaces" and $\mathbb{A}=$ "frames". Such situations are studied systematically in [18], and more recently also in the context of coalgebras and modal logic [21,20,5,6,19]. Typically the functor $P$ describes predicates on spaces and the functor $F$ theories of logical models.

In this situation it is important to keep track of the direction of arrows. To be explicit, the (components of the) unit and counit of the adjunction $F \dashv P$ are maps $\eta_{A}: A \rightarrow P F A$ in $\mathbb{A}$ and $\varepsilon_{X}: F P X \rightarrow X$ in $\mathbb{C}^{\text {op }}$, i.e. $\varepsilon_{X}: X \rightarrow F P X$ in $\mathbb{C}$. The familiar triangular identities are $P \varepsilon \circ \eta P=$ id in $\mathbb{A}$ and $\varepsilon F \circ F \eta=$ id in $\mathbb{C}^{\text {op }}$, i.e. $F \eta \circ \varepsilon F=\mathrm{id}$ in $\mathbb{C}$.

\subsection{Examples}

The following three instances of the dual adjunction (2) will be used throughout the paper. 


\section{Sets versus Boolean algebras}

The first dual adjunction is between sets and Boolean algebras:

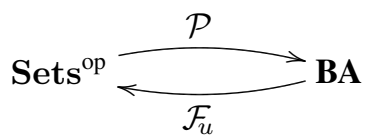

Here, BA is the category of Boolean algebras. The functor $\mathcal{P}$ is (contravariant) powerset and $\mathcal{F}_{u}$ sends a Boolean algebra $A$ to the set of its ultrafilters. These ultrafilters are filters (see below) $\alpha \subseteq A$ such that for each $a \in A$, either $a \in \alpha$ or $\neg a \in \alpha$, but not both. The unit $\eta_{A}: A \rightarrow \mathcal{P} \mathcal{F}_{u}(A)$ for this adjunction is given by $\eta(a)=\left\{\alpha \in \mathcal{F}_{u}(A) \mid a \in \alpha\right\}$. The adjunction (3) amounts to the standard correspondence:

$$
\frac{X \stackrel{f}{\longrightarrow} \mathcal{F}_{u}(A) \quad \text { in Sets }}{A \underset{g}{\longrightarrow} \mathcal{P}(X) \text { in BA }} \quad \text { via } \quad \frac{a \in f(x)}{\overline{x \in g(a)}}
$$

\section{Sets versus meet semilattices}

The second example uses the category MSL of meet semilattices, in a situation:

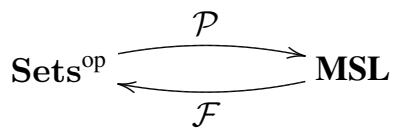

The functor $\mathcal{F}$ sends a meet semilattice $A$ to the set $\mathcal{F}(A)$ of its filters, i.e. to the upsets $\alpha \subseteq A$ which are closed under finite meets: $\top \in \alpha$ and $x, y \in \alpha \Rightarrow x \wedge y \in \alpha$. Here the unit is as before, $\eta_{A}(a)=\{\alpha \in \mathcal{F}(A) \mid a \in \alpha\}$, and the correspondence is also as before.

\section{Measure spaces versus meet semilattices}

Our third example is less standard. It uses the category Meas of measure spaces, instead of Sets. An object of Meas is a pair $\mathcal{X}=\left(X, S_{X}\right)$ of a set $X$ together with a $\sigma$ algebra $S_{X} \subseteq \mathcal{P}(X)$. The latter is a collection of "measurable" subsets closed under $\emptyset$, complements (negation), and countable unions. We shall use that it is closed, in particular, under finite intersections. A morphism $\mathcal{X} \rightarrow \mathcal{Y}$ in Meas, from $\mathcal{X}=\left(X, S_{X}\right)$ to $\mathcal{Y}=\left(Y, S_{Y}\right)$, is any measurable function $f: X \rightarrow Y$, i.e. a function satisfying $f^{-1}(M) \in S_{X}$ for each measurable set $M \in S_{Y}$.

Interestingly, in this case we also have an adjunction with meet semilattices:

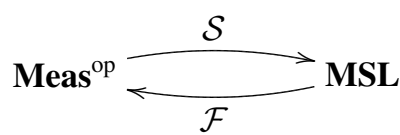


The functor $\mathcal{S}$ maps a measure space to its $\sigma$-algebra, i.e., for $\mathcal{X}=\left(X, S_{X}\right), \mathcal{S}(\mathcal{X})=$ $S_{X}$. The functor $\mathcal{F}$ is the filter functor from (4) that maps a meet semilattice to the set of its filters, with a $\sigma$-algebra generated by the subsets $\eta(a) \subseteq \mathcal{F}(A)$, for $a \in A$. Again we have a bijective correspondence

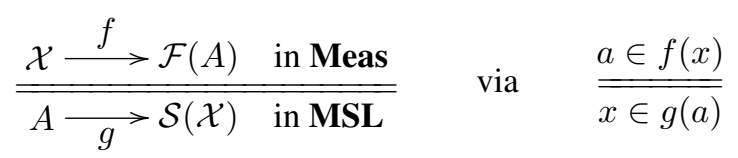

- Given a measurable function $f: X \rightarrow \mathcal{F}(A)$, we obtain $\widehat{f}: A \rightarrow \mathcal{S}(\mathcal{X})$ as:

$$
\widehat{f}(a)=f^{-1}(\eta(a))=\{x \in X \mid f(x) \in \eta(a)\}=\{x \in X \mid a \in f(x)\} .
$$

This $\widehat{f}$ is well-defined because $f$ is a measurable function, so $f^{-1}(\eta(a)) \in \mathcal{S}(\mathcal{X})$, and it preserves finite meets $T, \wedge$ because $\eta$ and $f^{-1}$ do.

- Conversely, given $g: A \rightarrow \mathcal{S}(\mathcal{X})$ in MSL one defines $\widehat{g}: X \rightarrow \mathcal{F}(A)$ as $\widehat{g}(x)=$ $\{a \in A \mid x \in g(a)\}$. This yields a filter because $g$ preserves finite meets. The function $\widehat{g}$ is measurable since $\widehat{g}^{-1}(\eta(a))=\{x \in X \mid a \in \widehat{g}(x)\}=g(a) \in \mathcal{S}(\mathcal{X})$.

It is obvious that $\widehat{\widehat{f}}=f$ and $\widehat{\widehat{g}}=g$. The unit $\eta_{A}: A \rightarrow \mathcal{S F}(A)$ of this adjunction is as before: $\eta_{A}(a)=\{\alpha \in \mathcal{F}(A) \mid a \in \alpha\}$.

Remark 1 In the end we notice that the adjunction Sets $^{o p} \leftrightarrows$ MSL can be obtained from the adjunction Meas ${ }^{o p} \leftrightarrows \mathbf{M S L}$ in the following manner. The forgetful functor $U:$ Meas $\rightarrow$ Sets, $U(\mathcal{X})=X$ for $\mathcal{X}=\left(X, S_{X}\right)$, has a left adjoint $D$ which equips a set $X$ with the discrete $\sigma$-algebra $\mathcal{P}(X)$ in which all subsets are measurable. Then, when we switch to opposite categories, the forgetful functor $U: \mathbf{M e a s}^{o p} \rightarrow$ Sets $^{o p}$ is left adjoint to $D$. Hence the adjunction $\mathcal{F} \dashv \mathcal{P}$ between sets and meet semilattices can be obtained as $U \mathcal{F} \dashv \mathcal{S} D$ by composition of adjoints in:

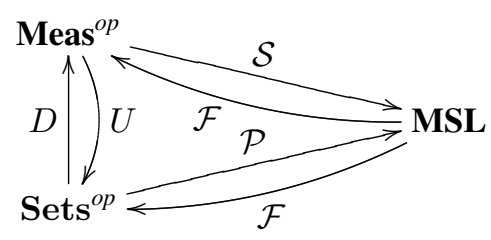

\section{Logical set-up}

We now extend the adjunction (2) with endofunctors $T$ and $L$ as in:

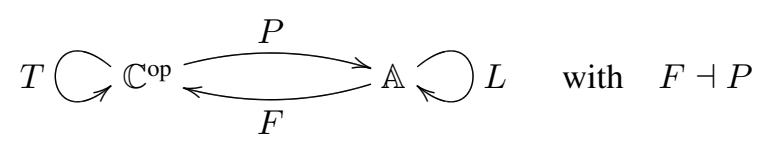

We shall be interested in coalgebras of the functor $T$, describing dynamic systems, and in algebras of $L$, capturing logical models. These models typically contain certain 
logical connectives, as incorporated in categories of Boolean algebras or of meet semilattices; the functor $L$ adds modal operators. Via a suitable relation between $T$ and $L$ one can capture logics for dynamic systems in this set-up. But before doing so we recall the following standard result.

Proposition 2 In the situation of the previous diagram we have a bijective correspondence between natural transformations:

$$
\frac{L P \stackrel{\sigma}{\Longrightarrow} P T}{\Longrightarrow F \rightleftharpoons \stackrel{\rightleftharpoons}{\rightleftharpoons} F L}
$$

i.e.

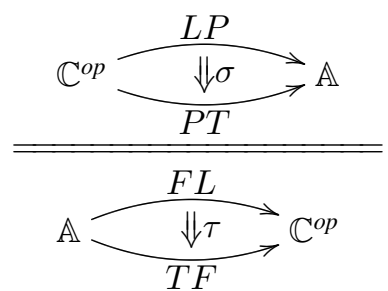

Proof. The correspondence is obtained as follows.

- For $\sigma: L P \Rightarrow P T$ one puts:

$$
\bar{\sigma} \stackrel{\text { def }}{=}(T F \stackrel{\varepsilon T F}{=} F P T F \stackrel{F \sigma F}{=} F L P F \stackrel{F L \eta}{=} F L)
$$

- Conversely, for $\tau: T F \Rightarrow F L$ one similarly takes:

$$
\bar{\tau} \stackrel{\text { def }}{=}(L P \stackrel{\eta L P}{=} P F L P \stackrel{P \tau P}{=} P T F P \stackrel{P T \varepsilon}{=} P T) .
$$

Assumption 3 In the situation (6) we shall assume the following.

- There is an "interpretation" natural transformation $\sigma: L P \Rightarrow P T$.

- The functor $L: \mathbb{A} \rightarrow \mathbb{A}$ has an initial algebra of "formulas". We shall write it as $L($ Form $) \cong$ Form.

- There is a factorisation system $(\mathcal{M}, \mathcal{E})$ on the category $\mathbb{C}$ with $\mathcal{M} \subseteq$ Monos and $\mathcal{E} \subseteq$ Epis. We shall write the maps in $\mathcal{M}$ as $\longmapsto$ and those in $\mathcal{E}$ as $\rightarrow$, and call them abstract monos and abstract epis, respectively. Hence every map in $\mathbb{C}$ factors as abstract mono-epi $\rightarrow \longmapsto$ and the "diagonal fill-in" property holds: if the outer square

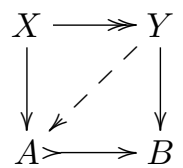

commutes, then there exists a diagonal map making the two triangles commute.

- The functor $T$ preserves the maps in $\mathcal{M}$, i.e. $m \in \mathcal{M} \Rightarrow T(m) \in \mathcal{M}$.

It is not hard to see that such a factorisation system lifts to the category $\operatorname{CoAlg}(T)$ of coalgebras of the functor $T$. Given a coalgebra homomorphism $(X \stackrel{c}{\longrightarrow} T X) \stackrel{f}{\longrightarrow}$ 
$(Y \stackrel{d}{\rightarrow} T Y)$ we can factorise $f=m \circ e$ in $\mathbb{C}$ and obtain a coalgebra $d^{\prime}$ by diagonal fill-in, since $T$ preserves abstract monos.

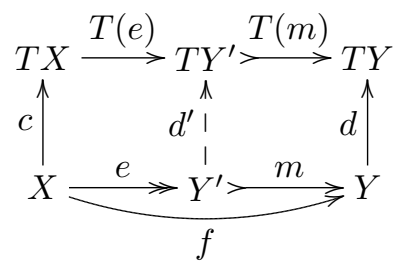

One can show that the diagonal fill-in property also holds in $\operatorname{CoA} \lg (T)$, using that $T$ preserves abstract monos, and abstract monos are monos.

For an arbitrary coalgebra $X \stackrel{c}{\rightarrow} T X$ by initiality of Form one obtains an interpretation of formulas as predicates on the state space $X$ as in:

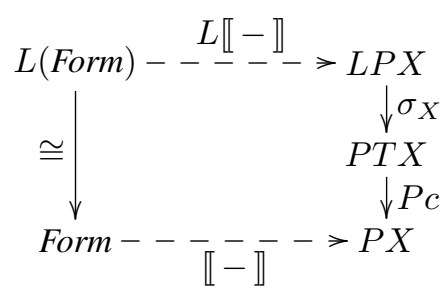

The adjunction $F \dashv P$ yields a theory map $t h: X \rightarrow F$ (Form) corresponding to the interpretation $\llbracket-\rrbracket:$ Form $\rightarrow P X$. Intuitively, for a state $x \in X$ we have a theory $\operatorname{th}(x) \in F($ Form $)$ of formulas that hold in $x$. Two states $x, y \in X$ will be called logically indistinguishable, written as $x \equiv y$, if their theories are the same: $\operatorname{th}(x)=$ $t h(y)$. In general, logical equivalence is the subobject of $X \times X$ which is the following equaliser in $\mathbb{C}$,

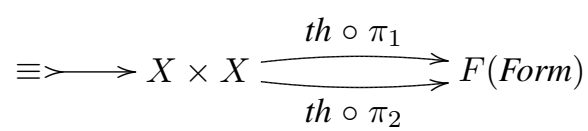

We are interested in comparing logical indistinguishability to behavioural equivalence. Two states $x, y \in X$ of a coalgebra are behaviourally equivalent, notation $x \approx y$, if there exists a coalgebra homomorphism $f$ with $f(x)=f(y)$. With the above assumptions, using (7), we may assume that this $f$ is an abstract epi.

Behavioural equivalence may also be formulated for two states $x \in X, y \in Y$ of two different coalgebras (of the same functor). One then requires that there exist coalgebra homomorphisms $f$ and $g$ with $f(x)=g(y)$. This formulation is equivalent to the previous one in categories with pushouts, see [28]. Behavioural equivalence coincides with bisimilarity in case the functor involved preserves weak pullbacks. In the context of expressivity of modal logics behavioural equivalence works better, as commonly accepted in the community, and noted explicitly for probabilistic systems in [8].

It is known and not difficult to show that behavioural equivalence implies logical indistinguishability. For the converse we now present our version of a "folklore" result (see also [19]). 
Theorem 4 In the context of Assumption 3, if the transpose $\bar{\sigma}: T F \Rightarrow F L$ of $\sigma: L P \Rightarrow$ $P T$, according to Proposition 2, is componentwise abstract mono, then logically indistinguishable elements are behaviourally equivalent.

Proof. One factors the theory map th: $X \rightarrow F$ (Form) as $X \stackrel{e}{\rightarrow} X^{\prime} \stackrel{m}{\longmapsto} F$ (Form). Then $x \equiv y$ if and only if $e(x)=e(y)$. The main point is to obtain a (quotient) coalgebra on $X^{\prime}$ via the diagonal fill-in property of the factorisation in:

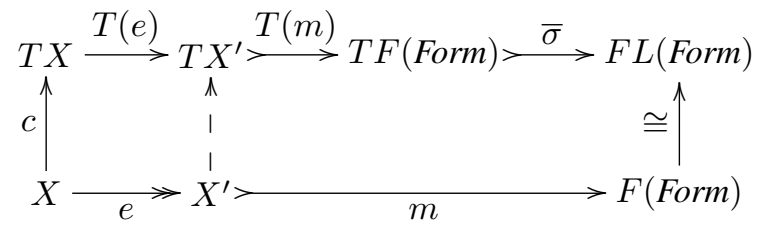

Logically indistinguishable elements are then equated by a coalgebra homomorphism (namely $e$ ), and are thus behaviourly equivalent.

The main technical part of applying Theorem 4 is showing that the natural transformation $\bar{\sigma}$ is mono. That will be the topic of the next section.

\subsection{Examples}

In the remainder of this section we extend the three adjunctions in the examples from Section 2 with suitable coalgebra functors - the $T$ in (6) - that we are interested in. Moreover, we discuss that Assumption 3 holds in each case. We consider two base categories, Sets and Meas. The category Sets has a standard factorisation system given by monos (injections) and epis (surjections), with a diagonal fill-in property. We discuss the factorisation system on Meas below in the section on Markov processes.

\section{Transition systems}

We shall write $\mathcal{P}_{f}:$ Sets $\rightarrow$ Sets for the finite powerset functor:

$$
\mathcal{P}_{f}(X)=\{S \subseteq X \mid S \text { is finite }\} .
$$

A coalgebra $X \rightarrow \mathcal{P}_{f}(X)$ is an image-finite unlabelled transition system. The functor $\mathcal{P}_{f}$ preserves injections.

\section{Multitransition systems}

Next we consider the finitely supported multiset functor $\mathcal{M}_{f}$ : Sets $\rightarrow$ Sets. It is described as follows.

$$
\mathcal{M}_{f}(X)=\{\varphi: X \rightarrow \mathbb{N} \mid \operatorname{supp}(\varphi) \text { is finite }\} .
$$

The support set of a multiset $\varphi$ is defined as $\operatorname{supp}(\varphi)=\{x \mid \varphi(x) \neq 0\}$. A function $f: X \rightarrow Y$ is mapped to $\mathcal{M}_{f}(f): \mathcal{M}_{f}(X) \rightarrow \mathcal{M}_{f}(Y)$ by

$$
\mathcal{M}_{f}(f)(\varphi)=\lambda y \in Y . \sum_{x \in f^{-1}(y)} \varphi(x) .
$$

A coalgebra $X \rightarrow \mathcal{M}_{f}(X)$ is a multitransition system in which multiple non-labelled transitions are possible between any two states. The functor $\mathcal{M}_{f}$ preserves injections. 


\section{Markov chains}

The third endofunctor on Sets is the finitely supported discrete subdistribution functor $\mathcal{D}_{f}:$ Sets $\rightarrow$ Sets. It is described as follows.

$$
\mathcal{D}_{f}(X)=\left\{\varphi: X \rightarrow[0,1] \mid \operatorname{supp}(\varphi) \text { is finite and } \sum_{x \in X} \varphi(x) \leq 1\right\} .
$$

The support set of a subdistribution $\varphi$ is defined, as before, as $\operatorname{supp}(\varphi)=\{x \mid \varphi(x) \neq$ $0\}$. A function $f: X \rightarrow Y$ yields a mapping $\mathcal{D}_{f}(f): \mathcal{D}_{f}(X) \rightarrow \mathcal{D}_{f}(Y)$ by

$$
\mathcal{D}_{f}(f)(\varphi)=\lambda y \in Y \cdot \sum_{x \in f^{-1}(y)} \varphi(x) .
$$

A coalgebra $X \rightarrow \mathcal{D}_{f}(X)$ is a Markov chain [33,3]. In this context subdistributions (with sum $\leq 1$ ) are more common than distributions (with sum $=1$ ), but the difference does not really matter here. The functor $\mathcal{D}_{f}$ preserves injections.

\section{What subsets, multisets, and distributions have in common}

Although they might seem different on first sight, the functors $\mathcal{P}_{f}, \mathcal{D}_{f}$, and $\mathcal{M}_{f}$ are all instances of the same generic functor. Let $(M,+, 0, \leq)$ be a partially ordered commutative monoid with the property

$$
x \leq x+y \quad \text { for all } x, y \in M .
$$

Let $O$ be any downward-closed subset of $M, O \subseteq M$ with $x \in O$ whenever $x \leq y, y \in$ $O$. Let $\mathcal{V}_{O}$ be the functor on Sets defined on objects as

$$
\mathcal{V}_{O}(X)=\left\{\varphi: X \rightarrow O \mid \operatorname{supp}(\varphi) \text { is finite and } \sum_{x \in X} \varphi(x) \in O\right\} .
$$

We will call the elements of $\mathcal{V}_{O}(X)$ valuations with values in $O$. The support set of a valuation is defined as before, $\operatorname{supp}(\varphi)=\{x \in X \mid \varphi(x) \neq 0\}$. Since $M$ is a commutative monoid with the property (9) and $O$ is downward-closed, any valuation $\varphi: X \rightarrow O$ extends to a function $\mathcal{P}(X) \rightarrow O$, which we also denote by $\varphi$, by

$$
\varphi(S)=\sum_{x \in S} \varphi(x) .
$$

Now, for a function $f: X \rightarrow Y$, we define $\mathcal{V}_{O}(f): \mathcal{V}_{O}(X) \rightarrow \mathcal{V}_{O}(Y)$ as

$$
\mathcal{V}_{O}(f)(\varphi)(y)=\left(\varphi \circ f^{-1}\right)(\{y\}) \quad \text { for } \quad \varphi \in \mathcal{V}_{O}(X), y \in Y .
$$

We have:

- subsets are valuations, $\mathcal{P}_{f}=\mathcal{V}_{O}$, for $M=(\{0,1\}, \vee, 0)$ with $O=M$.

- multisets are also valuations, $\mathcal{M}_{f}=\mathcal{V}_{O}$ for $M=(\mathbb{N},+, 0)$ also with $O=M$; and

- subdistributions are valuations as well, $\mathcal{D}_{f}=\mathcal{V}_{O}$ for $M=\left(\mathbb{R}^{\geq 0},+, 0\right)$ and $O=$ $[0,1]$. Notice that the requirement that the sum should be in $O=[0,1]$ automatically implies that the sum is at most one, as was required explicitly in the earlier description of Markov chains.

The difference between the functor $\mathcal{P}_{f}$, on the one hand, and both functors $\mathcal{M}_{f}$ and $\mathcal{D}_{f}$, on the other hand, is that the latter are instances of $\mathcal{V}_{O}$ for $O \subseteq M$ of cancellative monoids $M$, whereas $M=(\{0,1\}, \vee, 0)$ is not cancellative. This plays an important role for expressivity of the conjunction fragment of suitable modal logics, as we demonstrate below. 


\section{Markov processes}

On the category Meas we consider the Giry functor (or monad) from [12]. It maps a measure space $\mathcal{X}=\left(X, S_{X}\right)$ to the space $\mathcal{G}(\mathcal{X})=\left(\mathcal{G}_{\mathcal{X}}, \mathcal{S} \mathcal{G}(\mathcal{X})\right)$ of subprobability measures $\varphi: S_{X} \rightarrow[0,1]$, satisfying $\varphi(\emptyset)=0$ and $\varphi\left(\bigcup_{i} M_{i}\right)=\sum_{i} \varphi\left(M_{i}\right)$ for countable unions of pairwise disjoint subsets $M_{i} \in S_{X}$. For each $M \in S_{X}$ there is an evaluation function $e v_{M}: \mathcal{G}_{\mathcal{X}} \rightarrow[0,1]$ given by $\varphi \mapsto \varphi(M)$. The set $\mathcal{G}_{\mathcal{X}}$ is equipped with the smallest $\sigma$-algebra $\mathcal{S} \mathcal{G}(\mathcal{X})$ making all these maps $e v_{M}$ measurable. It is generated by the collection:

$$
\begin{aligned}
\left\{L_{r}(M) \mid r \in \mathbb{Q} \cap[0,1], M \in S_{X}\right\} \quad \text { where } \quad L_{r}(M) & =\left\{\varphi \in \mathcal{G}_{\mathcal{X}} \mid \varphi(M) \geq r\right\} \\
& =e v_{M}^{-1}([r, 1]) .
\end{aligned}
$$

These $L_{r}$ 's will be used later as modalities, see (19).

Let $\mathcal{X}=\left(X, S_{X}\right)$ and $\mathcal{Y}=\left(Y, S_{Y}\right)$ be measure spaces. On a measurable function $f: X \rightarrow Y$ one defines $\mathcal{G}(f): \mathcal{G}(\mathcal{X}) \rightarrow \mathcal{G}(\mathcal{Y})$, i.e., $\mathcal{G}(f): \mathcal{G}_{\mathcal{X}} \rightarrow \mathcal{G}_{\mathcal{Y}}$ by:

$$
\mathcal{G}(f)\left(S_{X} \stackrel{\varphi}{\rightarrow}[0,1]\right)=\left(S_{Y} \stackrel{f^{-1}}{\rightarrow} S_{X} \stackrel{\varphi}{\rightarrow}[0,1]\right) .
$$

This $\mathcal{G}(f)$ is a measurable function since for $M \in S_{Y}$ one has $\mathcal{G}(f)^{-1}\left(L_{r}(M)\right)=$ $L_{r}\left(f^{-1}(M)\right)$, where $f^{-1}(M) \in S_{X}$.

A coalgebra $\mathcal{X} \rightarrow \mathcal{G}(\mathcal{X})$ is a Markov process, see [10].

As factorisation system on Meas we take as abstract monos the collection $\mathcal{M}$ given by morphisms $f: \mathcal{X} \rightarrow \mathcal{Y}$ for which: $f$, as function $X \rightarrow Y$, is injective, and $f^{-1}$, as function $S_{Y} \rightarrow S_{X}$, is surjective. As abstract epis $\mathcal{E}$ we take those morphisms $f: \mathcal{X} \rightarrow$ $\mathcal{Y}$ for which $f$, as function $X \rightarrow Y$, is surjective. Every morphism $f: \mathcal{X} \rightarrow \mathcal{Y}$ in Meas factors as $X \stackrel{e}{\rightarrow} f(X) \stackrel{m}{\longmapsto} Y$ where the image $f(X)$ is given the $\sigma$-algebra $S_{f(X)}=\left\{m^{-1}(M) \mid M \in S_{Y}\right\}$. Clearly, $m^{-1}: S_{Y} \rightarrow S_{f(X)}$ is surjective. It is not hard to see that this factorisation system satisfies the diagonal fill-in property.

Finally we check that $\mathcal{G}$ preserves the maps in $\mathcal{M}$. Given a measurable function $f: X \rightarrow Y$ in $\mathcal{M}$, so that $f$ is injective and $f^{-1}: S_{Y} \rightarrow S_{X}$ is surjective, we claim that also $\mathcal{G}(f) \in \mathcal{M}$.

- The map $\mathcal{G}(f)$ is injective: Assume $\varphi, \psi \in \mathcal{G}_{\mathcal{X}}$ with $\mathcal{G}(f)(\varphi)=\mathcal{G}(f)(\psi)$, i.e. $\varphi$ o $f^{-1}=\psi \circ f^{-1}$. Since $f^{-1}$ is surjective, it can be canceled on the right and we get $\varphi=\psi$.

- The map $\mathcal{G}(f)^{-1}: \mathcal{S G}(\mathcal{Y}) \rightarrow \mathcal{S G}(\mathcal{X})$ is surjective: Let $L_{r}(M) \in \mathcal{S G}(\mathcal{X})$ be a generator, where $M \in S_{X}$ and $r \in \mathbb{Q} \cap[0,1]$. Since $f^{-1}$ is surjective we can find a measurable subset $N \in S_{Y}$ with $M=f^{-1}(N)$. But then $\mathcal{G}(f)^{-1}\left(L_{r}(N)\right)=$ $L_{r}\left(f^{-1}(N)\right)=L_{r}(M)$. This is enough to conclude surjectivity of $\mathcal{G}(f)^{-1}$ since all set operations are preserved by inverse images.

Remark 5 In Remark 1 we have used the (discrete) adjunction between sets and measure spaces in order to relate the adjunctions with meet semilattices. Here we shall also 
relate the subdistribution functor $\mathcal{D}_{f}$ and the Giry functor $\mathcal{G}$, in the situation:

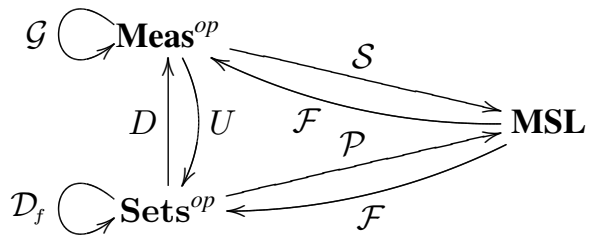

There is an obvious natural transformation $\rho: \mathcal{D}_{f} U \Rightarrow U \mathcal{G}$ with component on $\mathcal{X}=$ $\left(X, S_{X}\right) \in$ Meas, $\rho_{\mathcal{X}}: \mathcal{D}_{f}(X) \rightarrow \mathcal{G}_{\mathcal{X}}$ given by:

$$
\left[\begin{array}{r}
\varphi \in \mathcal{D}_{f}(X) \text { i.e. } \varphi: X \rightarrow[0,1] \text { with } \\
\operatorname{supp}(\varphi) \text { finite and } \sum_{x \in X} \varphi(x) \leq 1
\end{array}\right] \longmapsto\left[\begin{array}{l}
\text { the measure } S_{X} \rightarrow[0,1] \text { given by } \\
M \mapsto \varphi(M)=\sum_{x \in M} \varphi(x)
\end{array}\right]
$$

This $\rho$ captures the standard way in which a discrete measure (on points) forms a proper measure (on events/subsets). It plays an imporant role, later on in Section 4.4.

\section{Expressivity results}

The aim of this section is to show that the functors $\mathcal{P}_{f}, \mathcal{M}_{f}, \mathcal{D}_{f}$, and $\mathcal{G}$ given in the examples of Section 3 have expressive logics via Theorem 4. Thus we shall define, for each of them, an associated modality functor with interpretation natural transformation $\sigma$ whose transpose $\bar{\sigma}$ is componentwise (abstract) mono. We shall first describe the finite powerset $\mathcal{P}_{f}$ case because it is already well-studied, see $e . g$. [13,21,7]. This will set the scene for the other examples. Their expressivity is the main topic of this paper.

\subsection{Boolean logic for image-finite transition systems}

Expressivity of modal logic for image-finite transition systems has originally been proved in [13]. Such transition systems can be captured as coalgebras of the finite powerset functor $\mathcal{P}_{f}$. Coalgebraic generalisations of this expressivity result have been studied in e.g. [31,7].

Here we shall reproduce this expressivity result for $\mathcal{P}_{f}$ in the context of dual adjunctions, following [7]. We do so not only in order to prepare for the more complicated probabilistic examples $\mathcal{D}_{f}$ and $\mathcal{G}$ later on, but also to indicate where the negations of Boolean algebras are used. The main point of the latter examples $\mathcal{M}_{f}, \mathcal{D}_{f}$, and $\mathcal{G}$ is that they do not involve negation (nor disjunctions).

For a transition system $c: X \rightarrow \mathcal{P}_{f}(X)$ as coalgebra, the familiar modal operator $\square(c)$ is described as:

$$
\begin{aligned}
\square(c)(S) & =\{x \in X \mid \forall y \cdot x \rightarrow y \Rightarrow y \in S\} \\
& =\{x \in X \mid c(x) \subseteq S\} \\
& =c^{-1}(\square S),
\end{aligned}
$$


where $\square: \mathcal{P}(X) \rightarrow \mathcal{P}\left(\mathcal{P}_{f}(X)\right)$ is defined independently of the coalgebra $c$ as:

$$
\square(S)=\left\{u \in \mathcal{P}_{f}(X) \mid u \subseteq S\right\} .
$$

It is not hard to see that $\square$ preserves finite meets (intersections) and is thus a morphism in the category MSL. This leads to the following more abstract description.

Proposition 6 There is an endofunctor $L: \mathbf{B A} \rightarrow \mathbf{B A}$ in a situation:

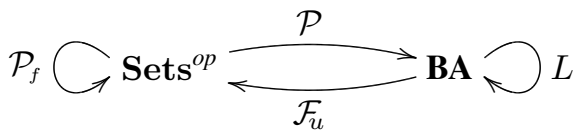

which is an instance of (6), such that the definition of $\square$ in (10) corresponds to an interpretation natural transformation $\otimes: L \mathcal{P} \Rightarrow \mathcal{P} \mathcal{P}_{f}$.

This shows that the familiar $\square$ operator fits into the current framework of dual adjunctions, so that we can use Theorem 4 . The details of the functor $L$ are not so relevant, but are given for reasons of completeness.

Proof. The construction of the functor $L$ (and $\otimes$ ) follows [21]. There is an obvious forgetful functor $V: \mathbf{B A} \rightarrow \mathbf{M S L}$, which has a left adjoint $G$. We write $L=G V: \mathbf{B A} \rightarrow$ BA for the resulting functor (actually comonad). By $\theta$ and $\xi$ we denote the unit and the counit of this adjunction, respectively. Notice that morphisms $L A \rightarrow B$ in BA correspond, via the adjunction, to functions $V A \rightarrow V B$ that preserve finite meets. The map $\square: \mathcal{P}(X) \rightarrow \mathcal{P} \mathcal{P}_{f}(X)$ from (10) is formally such a map $V \mathcal{P}(X) \rightarrow V \mathcal{P} \mathcal{P}_{f}(X)$ in the category MSL. Hence it corresponds to a map $\nabla: L \mathcal{P}(X) \rightarrow \mathcal{P} \mathcal{P}_{f}(X)$ as claimed. $\diamond$

Remark 7 On first sight it might seem that it is enough to take for $L$ the identity functor. However, this is not the case since each component of the interpretation natural transformation is required to be an arrow in $\mathbf{B A}$, whereas the modality maps $\square$ preserve finite intersections only.

The Boolean algebras $L A=G V A$ can be understood as models of Boolean logic with a finite meet preserving modal operator $\mathbf{\square}:$ We put $=\theta_{V A} \circ V\left(\xi_{A}\right): V L A \rightarrow$ $V L A$. The unit $\theta_{V A}: V A \rightarrow V L A$ of the adjunction $G \dashv V$ embeds elements (formulas) of a Boolean algebra $A$ into its extension $L A$ with -which is illustrated after the next result.

Lemma 8 The above defined finite meet preserving endofunction (or modal operator) satisfies:

1. idempotency: $\mathbf{\square} \circ \mathbf{\square}=\mathbf{\square}$

2. naturality: $V L f \circ \mathbf{\square}=\mathbf{\square} \circ L f$, for $f: A \rightarrow B$ in $\mathbf{B A}$;

3. $V \widehat{f} \circ \square=f \circ V \xi_{A}$ for $f: V A \rightarrow V B$ finite meet preserving, with $\widehat{f}: L A \rightarrow B$ as corresponding transpose. 
This last point of Lemma 8 yields a relation between the three boxes, as expressed by the following commuting diagram.

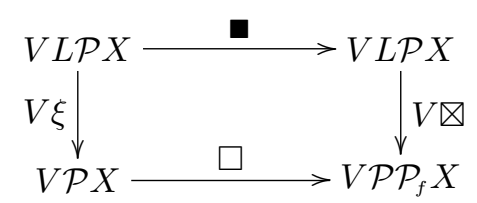

This diagram tells us that the operator is interpreted appropriately. This is best illustrated for the initial algebra $\alpha: L($ Form $) \cong$ Form with its interpretation map 【- \: Form $\rightarrow P X$ from (8), for a coalgebra $c: X \rightarrow T X$. Consider an arbitrary formula $\varphi \in$ Form and map it into $L$ (Form), formally by considering $\varphi \in V$ (Form) and applying $\theta(\varphi) \in V L($ Form $)$. Then we can apply and obtain $\theta(\varphi) \in V L($ Form $)$, which can be sent back to Form $=V($ Form $)$ via the initial algebra $\alpha$, resulting in $V(\alpha)(\boldsymbol{\square} \theta(\varphi)) \in V($ Form $)$. For convenience, this formula may simply be written as $\varphi$. We have been very explicit about all the maps and (forgetful) functors involved, so that we can check that this box-formula is interpreted appropriately:

$$
\begin{aligned}
& \llbracket \mathbf{\square} \varphi \rrbracket=(V \llbracket-\rrbracket \circ V \alpha \circ \mathbf{\square} \circ \theta)(\varphi) \\
& =(V P c \circ V \otimes \circ V L \llbracket-\rrbracket \circ \mathbf{\square} \circ \theta)(\varphi) \quad \text { by (8) } \\
& =(V P c \circ V \otimes \circ \mathbf{\square} \circ V L \llbracket-\rrbracket \circ \theta)(\varphi) \quad \text { by Lemma 8.(2) } \\
& =(V P c \circ \square \circ V(\xi) \circ \theta \circ V \llbracket-\rrbracket)(\varphi) \quad \text { by (11) and naturality } \\
& =(V P c \circ \square \circ V \llbracket-\rrbracket)(\varphi) \quad \text { by the triangular identities } \\
& =c^{-1}(\square \llbracket \varphi \rrbracket) \\
& =\{x \in X \mid \forall y . x \rightarrow y \Rightarrow y \in \llbracket \varphi \rrbracket\} \text {. }
\end{aligned}
$$

The next result now gives a semantical reformulation, following [7], of the expressivity result of [13]. It uses Theorem 4 . The proof that we present is substantially more complicated than the standard proof, but we include it in order to illustrate the general method on a well-known example. In the other applications the proofs actually become simpler than the original ones.

Theorem 9 The transpose $\bar{\nabla}: \mathcal{P}_{f} \mathcal{F}_{u} \Rightarrow \mathcal{F}_{u} L$ of $\bigotimes$ in Proposition 6, according to Proposition 2, is componentwise mono. Hence Boolean modal logic with $\square$ is expressive for image-finite transition systems.

Proof. By unraveling the definition of $\bar{\nabla}$ given in the proof of Proposition 2 we see, for finite $S \subseteq \mathcal{F}_{u} A$,

$$
\bar{\nabla}(S)=\{\varphi \in L A \mid S \in \otimes(L(\eta)(\varphi))\},
$$

where $\eta=\lambda a .\{\alpha \mid a \in \alpha\}$ is the unit $A \rightarrow \mathcal{P} \mathcal{F}_{u} A$ of the dual adjunction $\mathcal{F}_{u} \dashv \mathcal{P}$.

In order to prove injectivity of $\bar{\otimes}$, assume $S, M \in \mathcal{P}_{f} \mathcal{F}_{u}(A), S \neq M$, say $\alpha \in S$, $\alpha \notin M=\left\{\beta_{1}, \ldots, \beta_{n}\right\}$ for ultrafilters $\alpha, \beta_{i} \in \mathcal{F}_{u}(A)$. Then $\alpha \neq \beta_{i}$, so there are 
elements $b_{i} \in \beta_{i}$ with $b_{i} \notin \alpha$. These $b_{i}$ exist because we work in a Boolean algebra, with negation $\neg$, and each ultrafilter $\gamma$ satisfies either $a \in \gamma$ or $\neg a \in \gamma$, for each $a \in A$.

We can put these $b_{i}$ into $L A$ as $\theta\left(b_{i}\right)$, take their join and write $a=\mathbf{a}\left(\bigvee_{i} \theta\left(b_{i}\right)\right) \in$ $L A$. Then, for any set $W \in \mathcal{P}_{f} \mathcal{F}_{u}(A)$,

$$
\begin{aligned}
& a \in \bar{\nabla}(W) \Longleftrightarrow W \in \nabla\left(L(\eta)\left(\mathbf{\square} \bigvee_{i} \theta\left(b_{i}\right)\right)\right) \\
& \Longleftrightarrow W \in \otimes\left(\mathbf{\square} L(\eta)\left(\bigvee_{i} \theta\left(b_{i}\right)\right)\right) \quad \text { by } 2 \text {. in Lemma } 8 \\
& \Longleftrightarrow W \in \otimes\left(\boldsymbol{\square} \bigvee_{i} L(\eta)\left(\theta\left(b_{i}\right)\right)\right) \\
& \Longleftrightarrow W \in \otimes\left(\mathbf{\square} \bigvee_{i} \theta\left(\eta\left(b_{i}\right)\right)\right) \quad \text { by naturality } \\
& \Longleftrightarrow W \in \square\left(V(\xi) \bigvee_{i} \theta\left(\eta\left(b_{i}\right)\right)\right) \quad \text { via (11) } \\
& \Longleftrightarrow W \in \square\left(\bigcup_{i} V(\xi) \theta\left(\eta\left(b_{i}\right)\right)\right) \quad \text { since } \xi \text { is a map in BA } \\
& \Longleftrightarrow W \in \square\left(\bigcup_{i} \eta\left(b_{i}\right)\right) \quad \text { by the triangular equations } \\
& \Longleftrightarrow W \subseteq \bigcup_{i} \eta\left(b_{i}\right) \\
& \Longleftrightarrow \forall \alpha \in W . \exists i . b_{i} \in \alpha \text {. }
\end{aligned}
$$

Then $a \in \bar{\nabla}(M)$, but $a \notin \bar{\nabla}(S)$, proving that $\bar{\nabla}$ is mono.

Remark 10 As is well-known, the finite powerset $\mathcal{P}_{f}$ is a monad on Sets and hence a comonad on Sets $^{o p}$. The functor L: BA $\rightarrow$ BA in Proposition 6 is a comonad by construction. We do not use these comonad structures in this paper but we do like to point out that they are related in the following sense: the functor $\mathcal{P}:$ Sets $^{o p} \rightarrow \mathbf{B A}$ with natural transformation $\otimes: L \mathcal{P} \Rightarrow \mathcal{P} \mathcal{P}_{f}$ is a morphism of comonads. Hence there is a close connection between the two sides of the dual adjunction in Proposition 6.

The underlying reason is that the natural transformation $\square: \mathcal{P} \Rightarrow \mathcal{P} \mathcal{P}_{f}$ from (10) commutes with the monad operations $\iota=\{-\}, \mu=\bigcup$ of the monad $\mathcal{P}_{f}$, in the sense that the following diagram commutes (in MSL).

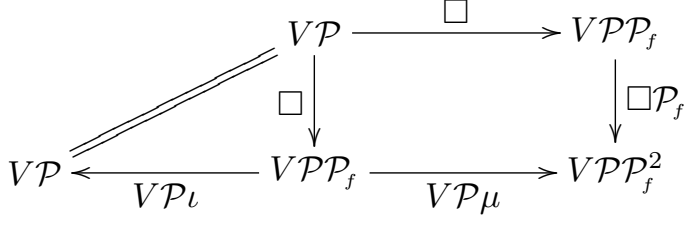

Hence, $\mathcal{P}$ with $\otimes$ indeed constitutes a comonad morphism. As a consequence we get, for example, the result:

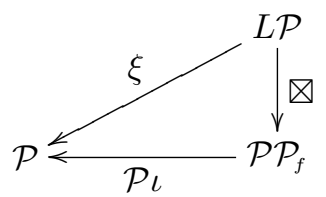

since:

$$
\mathcal{P} \iota \circ \otimes=\mathcal{P} \iota \circ \xi \circ G(\square)=\xi \circ G V \mathcal{P} \iota \circ G(\square)=\xi
$$

Similarly for commutation of $\otimes$ with (co)multiplications. 


\subsection{Finite-conjunctions logic for multitransition systems and Markov chains}

The situation for the finitely supported multiset functor and the finitely supported distribution functor $\mathcal{D}_{f}$ is similar, but a bit more complicated. We obtain an expressivity result for finitely supported cancellative "valuation" systems and logic with the corresponding modalities and only finite conjunctions. This result then directly instantiates both to multitransition systems and to Markov chains.

It has been shown in [7] that Boolean logic together with probabilistic modalities is expressive for Markov chains. That result also fits in the framework of dual adjunctions [7]. Here we provide an expressivity result for a weaker logic, namely with finite conjunctions only. It has also been shown that graded modal logic is expressive for multitransition systems [31]. Now we show that finite conjunctions suffice in this case as well.

We focus on the valuations functor $\mathcal{V}_{O}$ for a subset $O \subseteq M$ of an ordered cancellative monoid $(M,+, 0)$, with the property $x \leq x+y$ for all $x, y \in M$, as defined in Section 3.1. A subset $\widehat{O} \subseteq O$ is dense in $O$ if between any two elements $x, y \in O$ with $x \leq y$, there exists an element $z \in \widehat{O}$ such that $x \leq z \leq y$. Note that $O$ is dense in itself.

For a dense subset $\widehat{O}$ of $O$, we consider the "valuation modalities" $\square_{o}: \mathcal{P}(X) \rightarrow$ $\mathcal{P} \mathcal{V}_{O}(X)$, for $o \in \widehat{O}$, given as:

$$
\square_{o}(S)=\left\{\varphi \in \mathcal{V}_{O}(X) \mid \sum_{s \in S} \varphi(x) \geq o\right\},
$$

From (9) it follows that $\square_{o}$ is a monotone function, and thus a map in the category PoSets of posets and monotone functions. From these $\square_{o}$ we get a modality functor like in Proposition 6, bringing us in the framework of dual adjunctions.

Proposition 11 There is an endofunctor $K_{\widehat{O}}:$ MSL $\rightarrow$ MSL in a situation:

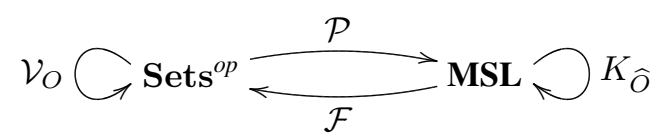

which is an instance of (6), such that all $\square_{o}$ in (12) correspond to an interpretation natural transformation $\Downarrow: K_{\widehat{O}} \mathcal{P} \Rightarrow \mathcal{P} \mathcal{V}_{O}$.

Proof. In order to construct $K_{\widehat{O}}$, we now consider the forgetful functor $V:$ MSL $\rightarrow$ PoSets with its left adjoint $H$. As before, we denote the unit and the counit of this adjunction by $\theta$ and $\xi$, respectively. We then define the functor $K_{\widehat{O}}:$ MSL $\rightarrow$ MSL as:

$$
K_{\widehat{O}}(A)=\coprod_{o \in \widehat{O}} H V A
$$

Here we use that the category MSL has arbitrary coproducts—which follows for instance from Linton's Theorem (see [2]), using that MSL is algebraic over Sets (and thus cocomplete). A map $K_{\widehat{O}}(A) \rightarrow B$ in MSL now corresponds to an $\widehat{O}$ indexed family of (monotone) functions $V A \rightarrow V B$ in PoSets. The family of maps $\square_{o}: \mathcal{P}(X) \rightarrow \mathcal{P} \mathcal{V}_{O}(X)$ from (12) is formally such an $\widehat{O}$-indexed family of functions $V \mathcal{P}(X) \rightarrow V \mathcal{P} \mathcal{V}_{O}(X)$ in PoSets. Hence, this family corresponds to a (natural) map $\bigotimes: K_{\widehat{O}} \mathcal{P}(X) \rightarrow \mathcal{P} \mathcal{V}_{O}(X)$. 
As before, the meet semilattices $K_{\widehat{O}}(A)$ can be seen as models of logic with only finite conjunctions, with a family of order preserving modal operators $\mathbf{m}_{o}: V K_{\widehat{O}}(A) \rightarrow$ $V K_{\widehat{O}}(A)$, for $o \in \widehat{O}$, defined as composite:

$$
V\left(\coprod_{o} H V A\right) \stackrel{V(\nabla)}{\longrightarrow} V H V A \stackrel{V\left(\xi_{A}\right)}{\longrightarrow} V A \stackrel{\theta_{V A}}{\longrightarrow} V H V A \stackrel{V\left(\kappa_{o}\right)}{\longrightarrow} V\left(\coprod_{o} H V A\right)
$$

where $\kappa_{o}$ is a coprojection and $\nabla=[\mathrm{id}]_{o}$ is the cotuple of identities. These monotone modal operators satisfy idempotency and naturality, and commute appropriately with $\square$ and $\otimes$. We do not elaborate on these $\mathbf{a}_{o}$ 's because we do not need them (explicitly) in the expressivity proof below.

Recall that the unit of the filter-powerset adjunction $\eta: A \rightarrow \mathcal{P F}(A)$, given by $\eta(a)=\{\alpha \in \mathcal{F} A \mid a \in \alpha\}$, preserves finite meets. We define for an arbitrary subset $\alpha \subseteq A$ the set of filters $\uparrow \alpha=\{\beta \in \mathcal{F} A \mid \alpha \subseteq \beta\}$ that contain $\alpha$. This map $\uparrow: \mathcal{P}(A) \rightarrow$ $\mathcal{P} \overline{\mathcal{F}}(A)$ can be seen as free extension of $\eta$ from $A$ to the complete lattice $(\mathcal{P}(A), \supseteq)$, since:

$$
\uparrow \alpha=\{\beta \in \mathcal{F} A \mid \forall a \in \alpha . a \in \beta\}=\bigcap_{a \in \alpha} \eta(a) .
$$

As a result $\alpha \subseteq \alpha^{\prime}$ implies $\uparrow \alpha \supseteq \uparrow \alpha^{\prime}$. We further note that:

$$
\begin{aligned}
\uparrow\left\{a_{1}, \ldots, a_{n}\right\} & =\left\{\beta \in \mathcal{F} A \mid a_{1}, \ldots, a_{n} \in \beta\right\} \\
& =\left\{\beta \in \mathcal{F} A \mid a_{1} \wedge \cdots \wedge a_{n} \in \beta\right\} \\
& =\eta\left(a_{1} \wedge \cdots \wedge a_{n}\right) .
\end{aligned}
$$

The next auxiliary property will be used for showing expressivity.

Lemma 12 Let $X$ be a set, $S \subseteq \mathcal{P}(X)$, and let $S_{f} \subseteq S$ be finite. Let $\uparrow_{S} \alpha=\{\beta \in S \mid$ $\forall a \in \alpha . a \in \beta\}$, for $\alpha \in \mathcal{P}(X)$. Then for each $\alpha \in \mathcal{P}(X)$ there is a finite $\alpha_{f} \subseteq \alpha$ with $S_{f} \cap \uparrow_{S} \alpha=S_{f} \cap \uparrow_{S} \alpha_{f}$

Proof. Write $S_{f}-\uparrow_{S} \alpha=\left\{\beta_{1}, \ldots, \beta_{n}\right\}$, where by construction $\alpha \nsubseteq \beta_{i}$, say via elements $a_{i} \in \alpha, a_{i} \notin \beta_{i}$. Take $\alpha_{f}=\left\{a_{1}, \ldots, a_{n}\right\} \subseteq \alpha$. Then $\uparrow_{S} \alpha_{f} \supseteq \uparrow_{S} \alpha$ and so $S_{f} \cap \uparrow_{S} \alpha_{f} \supseteq S_{f} \cap \uparrow_{S} \alpha$. For the converse, assume $\beta \in S_{f} \cap \uparrow_{S} \alpha_{f}$. If $\beta \notin \uparrow_{S} \alpha$, there must be an $i$ with $\beta=\beta_{i}$ and thus $a_{i} \notin \beta$. This contradicts $\beta \in \uparrow_{S} \alpha_{f}$.

We will apply the above lemma to semilattices $A$ with $S=\mathcal{F}(A)$, in which case $\uparrow_{S}=\uparrow$, as defined above. We next state and prove the expressivity result for logic with finite conjunctions and finitely supported valuation systems. It uses Theorem 4.

Theorem 13 The transpose $\bar{\nabla}: \mathcal{V}_{O} \mathcal{F} \Rightarrow \mathcal{F} K_{\widehat{O}}$ of $\otimes$ in Proposition 11, according to Proposition 2, is componentwise mono. Hence modal logic with finite conjunctions and valuation modalities is expressive for finitely supported valuation systems.

Proof. The transpose $\bar{\nabla}: \mathcal{V}_{O} \mathcal{F}(A) \rightarrow \mathcal{F} K_{\widehat{O}}(A)$ is given on a valuation $\Phi: \mathcal{F}(A) \rightarrow O$ on filters of $A$ as:

$$
\bar{\nabla}(\Phi)=\left\{\varphi \in K_{\widehat{O}}(A) \mid \Phi \in \bigotimes_{\mathcal{F}(A)}\left(K_{\widehat{O}}(\eta)(\varphi)\right)\right\} .
$$


We first note that for an element $a \in V A$ we have $\theta(a) \in V H V A$ and thus $a_{o}=V\left(\kappa_{o}\right)(\theta(a)) \in V K_{\widehat{O}}(A)$, for $o \in \widehat{O}$. For such elements $a_{o}$ we reason as follows, writing forgetful functors $V:$ MSL $\rightarrow$ PoSets explicitly in order to justify all manipulations.

$$
\begin{aligned}
& a_{o}= V\left(\kappa_{o}\right)(\theta(a)) \in V(\bar{\nabla})(\Phi) \\
& \Longleftrightarrow \Phi \in\left(V\left(\bigotimes \circ K_{\widehat{O}}(\eta)\right) \circ V\left(\kappa_{o}\right) \circ \theta\right)(a) \\
& \Longleftrightarrow \Phi \in\left(V\left(\left[\xi \circ H\left(\square_{p}\right)\right]_{p} \circ\left[\kappa_{p} \circ H V(\eta)\right]_{p} \circ \kappa_{o}\right) \circ \theta\right)(a) \\
& \quad \quad \text { by definition of } \bigotimes \text { and } K_{\widehat{O}} \\
& \Longleftrightarrow \Phi \in\left(V\left(\left[\xi \circ H\left(\square_{p}\right)\right]_{p} \circ \kappa_{o} \circ V H V(\eta) \circ \theta\right)(a)\right. \\
& \Longleftrightarrow \Phi \in\left(V(\xi) \circ V H\left(\square_{o}\right) \circ \theta \circ V(\eta)\right)(a) \\
& \Longleftrightarrow \Phi \in\left(V(\xi) \circ \theta \circ \square_{o} \circ V(\eta)\right)(a) \\
& \Longleftrightarrow \Phi \in\left(\square_{o} \circ V(\eta)\right)(a) \\
& \Longleftrightarrow \sum_{\alpha \in \eta(a)} \Phi(\alpha) \geq o \\
& \Longleftrightarrow \Phi(\eta(a)) \geq o .
\end{aligned}
$$

Towards injectivity of $\bar{\nabla}$ assume $\bar{\nabla}(\Phi)=\bar{\nabla}(\Psi)$. By the reasoning above we then get $\Phi(\eta(a)) \geq o \Leftrightarrow \Psi(\eta(a)) \geq o$ for all $o \in \widehat{O}$ and all $a \in A$. This yields:

$$
\Phi(\eta(a))=\Psi(\eta(a)) .
$$

Namely, since $\widehat{O}$ is dense in $O$, we have that for any $x, y \in O, \forall o \in \widehat{O} \cdot x \geq o \Longleftrightarrow y \geq$ $o$ implies $\forall o \in O . x \geq o \Longleftrightarrow y \geq o$, which further implies (taking $o=x$ and $o=y$ ) that $x=y$.

Let $S_{f}=\operatorname{supp}(\Phi) \cup \operatorname{supp}(\Psi)$ be the joined support of $\Phi$ and $\Psi$. Since $\Phi$ and $\Psi$ have finite support, $S_{f}$ is also finite. We now reach our second conclusion: for all filters $\alpha \in \mathcal{F} A$,

$$
\begin{array}{rlrl}
\Phi(\uparrow \alpha) & =\Phi\left(S_{f} \cap \uparrow \alpha\right) & & \text { since values outside } S_{f} \text { do not contribute to the sum } \\
& =\Phi\left(S_{f} \cap \uparrow \alpha_{f}\right) & \text { with } \alpha_{f} \subseteq \alpha \text { finite, as in Lemma } 12 \\
& =\Phi\left(\uparrow \alpha_{f}\right) & & \\
& =\Phi\left(\eta\left(\bigwedge \alpha_{f}\right)\right) & & \text { by (13), since } \alpha_{f} \text { is finite } \\
& =\Psi\left(\eta\left(\bigwedge \alpha_{f}\right)\right) & & \text { by (14) } \\
& =\ldots & & \text { as before } \\
& =\Psi(\uparrow \alpha) . &
\end{array}
$$

If $\Phi \neq \Psi$ we can now construct a contradiction: let $\alpha$ be a maximal filter with the property $\Phi(\alpha) \neq \Psi(\alpha)$. Such a maximal filter exists since $\Phi$ and $\Psi$ have finite support. Thus $\Phi(\beta)=\Psi(\beta)$ for $\beta \supsetneq \alpha$. But then, since the monoid is cancellative by 
assumption, we get:

$$
\begin{aligned}
\Phi(\uparrow \alpha) & =\Phi(\alpha)+\sum_{\beta \supsetneq \alpha} \Phi(\beta) \\
& =\Phi(\alpha)+\sum_{\beta \supsetneq \alpha} \Psi(\beta) \\
& \neq \Psi(\alpha)+\sum_{\beta \supsetneq \alpha} \Psi(\beta) \\
& =\Psi(\uparrow \alpha),
\end{aligned}
$$

contradicting (15).

As a consequence we get expressivity for multitransition systems and Markov chains, which we elaborate next. Recall that multitransition systems are $\mathcal{V}_{O}$-coalgebras for $M=O=\mathbb{N}$ which is a cancellative monoid (with $0,+$ ). The modalities $\nabla_{k}$ of graded modal logic, for $k \in \mathbb{N}$, are given by

$$
\diamond_{k}(S)=\left\{\varphi \in \mathcal{M}_{f}(X) \mid \sum_{s \in S} \varphi(x) \geq k\right\},
$$

and are obviously the valuation modalities for $\widehat{O}=O=\mathbb{N}$. Therefore we get the following result.

Corollary 14 The conjunction fragment of graded modal logic is expressive for multitransition systems.

Markov chains are $\mathcal{V}_{O}$-coalgebras for the cancellative monoid $M=\left(\mathbb{R}^{\geq 0},+, 0\right)$ with $O=[0,1]$. We consider the dense subset $\widehat{O}=\mathbb{Q} \cap[0,1]$ of $O$. The standard probabilistic modalities $L_{r}: \mathcal{P}(X) \rightarrow \mathcal{P} \mathcal{D}_{f}(X)$ are defined as:

$$
L_{r}(S)=\left\{\varphi \in \mathcal{D}_{f}(X) \mid \sum_{s \in S} \varphi(x) \geq r\right\},
$$

for $r \in \mathbb{Q} \cap[0,1]$, so they coincide with the valuation modalities. Hence we get expressivity for Markov chains.

Corollary 15 Finite conjunction modal logic with the standard probabilistic modalities is expressive for Markov chains.

Remark 16 In the expressivity proof of Theorem 13, a crucial point is the inequality (16), which fails for ordinary transition systems since the Boolean disjunction monoid $(\{0,1\}, \vee, 0)$ is not cancellative.

\subsection{Finite-conjunctions logic for Markov processes}

We now present an expressivity result for general, non-discrete, probabilistic systems and logic with the standard modalities and only finite conjunctions. This expressivity result was first shown in $[9,10]$ for Markov processes over analytic spaces, and recently for general Markov processes over any measure space [8]. It is common in the categorial treatment of non-discrete probabilistic systems $(c f .[11,9,10])$ to make the detour through analytic or Polish spaces. The main reason is that bisimilarity (in terms of spans) can not be described in general measure spaces, due to non-existence of pullbacks. However, as we already noted before, behavioural equivalence can, which is also 
one of the main points of [8] where an explicit characterisation of behavioural equivalence under the name event bisimulation is given. Hence, we consider general measure spaces.

We start from the general probabilistic modalities $L_{r}: \mathcal{S}(\mathcal{X}) \rightarrow \mathcal{S G}(\mathcal{X})$, where $\mathcal{X}=$ $\left(X, S_{X}\right)$, for $r \in \mathbb{Q} \cap[0,1]$.

$$
L_{r}(M)=\left\{\varphi \in \mathcal{G}_{\mathcal{X}} \mid \varphi(M) \geq r\right\},
$$

Note that these modalities are well-defined i.e. $L_{r}(M) \in \mathcal{S G}(\mathcal{X})$ by definition.

These modalities are obviously monotone. Hence we can use the functor $K=$ $K_{\widehat{O}}=\amalg_{o \in \widehat{O}} H V A$ for $\widehat{O}=\mathbb{Q} \cap[0,1]$ from Proposition 11. With this functor $K:$ MSL $\rightarrow$ MSL we transform these $L_{r}: V \mathcal{S}(\mathcal{X}) \rightarrow V \mathcal{S G}(\mathcal{X})$ in PoSets into a natural transformation $\otimes: K \mathcal{S} \Rightarrow \mathcal{S G}$ in the situation:

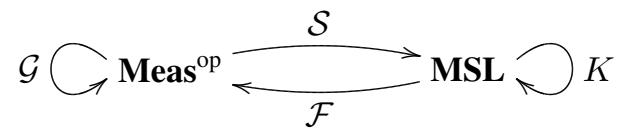

This means that $\otimes$ satisfies $V(\otimes) \circ V\left(\kappa_{r}\right) \circ \theta=L_{r}$, where $\theta: \operatorname{Id} \Rightarrow V H$ is the unit of the adjunction between MSL and PoSets.

We can now present the expressivity result for logic with finite conjunctions and finitely supported probabilistic systems (from [10,8]), using Theorem 4. It uses standard measure theoretic results [4], just like the proof in [10] does.

Theorem 17 The transpose $\bar{\nabla}: \mathcal{G F} \Rightarrow \mathcal{F} K$ of $₫$ described above, according to Proposition 2, is componentwise abstract mono. Hence modal logic with finite conjunctions and probabilistic modalities is expressive for Markov processes.

Proof. The transpose $\bar{\nabla}: \mathcal{G F}(A) \rightarrow \mathcal{F} K(A)$ is given, on a measure $\Phi: \mathcal{F}(A) \rightarrow[0,1]$ on filters of $A$, as:

$$
\bar{\nabla}(\Phi)=\left\{\varphi \in K A \mid \Phi \in \otimes_{\mathcal{F}(A)}(K(\eta)(\varphi))\right\} .
$$

As before, $\bar{\nabla}(\Phi)=\bar{\nabla}(\Psi)$ implies $\Phi(\eta(a))=\Psi(\eta(a))$ for all $a \in A$. Now we use [4, Theorem 10.4]: Let $\nu_{1}, \nu_{2}$ be finite measures on a measure space $\left(X, S_{X}\right)$ with generated $\sigma$-algebra $S_{X}$. If (1) the set of generators is closed under binary intersections, (2) the whole space $X$ is a countable union of generators, and (3) $\nu_{1}$ and $\nu_{2}$ coincide on the generators, then $\nu_{1}=\nu_{2}$. In our case $\Phi, \Psi$ are finite measures on $\mathcal{F}(A)$ with its $\sigma$-algebra $\mathcal{S F}(A)$ generated by the sets $\eta(a)$, for $a \in A$. The whole space $\mathcal{F}(A)$ is $\eta(\top)$ since $\top$ is a top element in $A$ which is contained in every filter. Moreover, $\Phi$ and $\Psi$ coincide on the generators. Hence, $\Phi=\Psi$ which shows that $\bar{\nabla}$ is a mono.

We still need to show that it satisfies the second condition for an abstract mono in Meas, namely that $\bar{\nabla}^{-1}: \mathcal{S F} K(A) \rightarrow \mathcal{S G \mathcal { F }}(A)$ is a surjective map. For a generator $L_{r}(N) \in \mathcal{S G F}(A)$, where $N \in \mathcal{S F}(A)$, we need to find a measurable set $M \in \mathcal{S F} K A$ with $\bar{\bigotimes}^{-1}(M)=L_{r}(N)$. We shall do so first for generators $N=\eta(a)$, for each $r \in$ $\mathbb{Q} \cap[0,1]$. 
Assume $N=\eta(a)=\{\alpha \in \mathcal{F}(A) \mid a \in \alpha\}$, for some $a \in A$. We then take $a_{r}=\left(\kappa_{r} \circ \theta\right)(a) \in K A$, or more formally, $a_{r}=V\left(\kappa_{r}\right)\left(\theta_{V A}(a)\right) \in V K A$ as in:

$$
a \in V A \stackrel{\theta_{V A}}{\longrightarrow} V H V A \stackrel{V\left(\kappa_{r}\right)}{\longrightarrow} V\left(\coprod_{r \in \mathbb{Q} \cap[0,1]} H V A\right)=V K A
$$

The measurable set $\eta\left(a_{r}\right) \in \mathcal{S F} K A$ does the job in this case:

$$
\begin{array}{rlr}
\bar{\nabla}^{-1}\left(\eta\left(a_{r}\right)\right) & =\left\{\Phi \mid \bar{\nabla}(\Phi) \in \eta\left(a_{r}\right)\right\} & \\
& =\left\{\Phi \mid a_{r} \in \bar{\nabla}(\Phi)\right\} & \\
& =\left\{\Phi \mid \Phi \in \bigotimes\left(K(\eta)\left(a_{r}\right)\right)\right\} & \\
& =\bigotimes\left(K(\eta)\left(\left(\kappa_{r} \circ \theta\right)(a)\right)\right) & \\
& =\bigotimes\left(\left(\kappa_{r} \circ \theta\right)(\eta(a))\right) & \text { by naturality } \\
& =L_{r}(\eta(a)) & \text { by definition of } \otimes .
\end{array}
$$

We will next show that the $\sigma$-algebra $\Sigma_{0}$ generated by the sets $L_{r}(\eta(a))$ equals the $\sigma$-algebra generated by $L_{r}(M)$ for arbitrary $M \in \mathcal{S F} A$, which we will denote simply

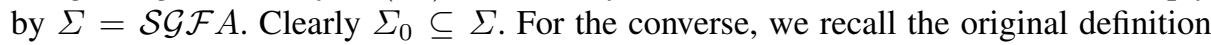
of $\Sigma$ : the smallest $\sigma$-algebra making the evaluation maps $e v_{M}$ measurable. We aim at showing that $\Sigma_{0}$ also makes all evaluation maps measurable, which completes the proof.

We start by defining the notion of $\lambda$-system. A collection of subsets $\Lambda \subseteq \mathcal{P}(X)$ is called a $\lambda$-system if (1) $X \in \Lambda$, (2) $M \in \Lambda \Longrightarrow \neg M \in \Lambda$, and (3) $\bigcup_{n} M_{n} \in \Lambda$, for all pairwise disjoint $M_{n} \in \Lambda$ where $n \in \mathbb{N}$.

We will further use the following result from [4, Theorem 3.2]. If $\mathcal{N} \subseteq \mathcal{P}(X)$ is closed with respect to binary intersections, then $\langle\mathcal{N}\rangle_{\lambda}=\langle\mathcal{N}\rangle_{\sigma}$, where $\langle\mathcal{N}\rangle_{\lambda}$ and $\langle\mathcal{N}\rangle_{\sigma}$ are the smallest $\lambda$-system and the smallest $\sigma$-algebra containing $\mathcal{N}$, respectively.

In our case, let $\mathcal{N}=\{\eta(a) \mid a \in A\}$, which is closed under finite intersections, and let

$$
\mathcal{L}=\left\{M \in \Sigma=\mathcal{S F} \mathcal{F} \mid e v_{M}:\left(\mathcal{G}_{\mathcal{F} A}, \Sigma_{0}\right) \rightarrow[0,1] \text { is measurable }\right\} .
$$

Note that all evaluation maps $e v_{M}$ are measurable on the measure space $\left(\mathcal{G}_{\mathcal{F} A}, \Sigma\right)$. In $\mathcal{L}$ we gather those $M$ such that $e v_{M}$ are measurable on the "smaller" measure space $\left(\mathcal{G}_{\mathcal{F A}}, \Sigma_{0}\right)$. We have that $\eta(a) \in \mathcal{L}$ for all $a \in A$, and the following hold:

(1) $\mathcal{F} A=\eta(\top) \in \mathcal{L}$;

(2) For $M \in \mathcal{L}$, we have that $\neg M=\mathcal{F} A \backslash M$ where $M \subseteq \mathcal{F} A$ and therefore $e v_{\neg M}=$ $e v_{\mathcal{F} A}-e v_{M}$. Then $e v_{\neg M}$ is a measurable map since measurable maps form a vector space, $c f$. [4, Theorem 13.3]. Hence $\neg M \in \mathcal{L}$.

(3) Let $M_{n} \in \mathcal{L}$ for $n \in \mathbb{N}$ be pairwise disjoint sets and let $M=\bigcup_{n} M_{n}$. Then, by the sigma-additivity of the measures, we have $e v_{M}(\varphi)=\sum_{n} e v_{M_{n}}(\varphi)$. We further consider the functions $e v_{M}^{k}=\sum_{n<k} e v_{M_{n}}$, which are measurable since measurable functions form a vector space. This sequence of functions is pointwise convergent, and therefore we can use [4, Theorem 13.4]: for any sequence of measurable functions with a pointwise limit, the limit function is measurable, to conclude that $e v_{M}$ is a measurable function, and therefore $M \in \mathcal{L}$. 
Hence, the set $\mathcal{L}$ is a $\lambda$-system and it contains the set $\mathcal{N}=\{\eta(a) \mid a \in A\}$. By the above mentioned theorem we get that $\langle\mathcal{N}\rangle_{\lambda}=\langle\mathcal{N}\rangle_{\sigma}=\mathcal{S} \mathcal{F} A$. Since $\mathcal{N} \subseteq \mathcal{L}$ and $\mathcal{L}$ is a $\lambda$-system, we get that $\langle\mathcal{N}\rangle_{\lambda} \subseteq \mathcal{L}$, which implies that $\mathcal{L}=\mathcal{S F} A$ and completes the proof.

\subsection{Relating Markov chains and Markov processes}

In Remarks 1 and 5 we have already seen how the categories and functors for Markov chains and for Markov processes are related. Here we elaborate further on these relations and complete the picture by showing how to obtain expressivity for chains from expressivity for processes. This depends on auxiliary results which are of interest on their own.

Recall that Markov chains and Markov processes are related via the natural transformation $\rho: \mathcal{D}_{f} U \Rightarrow U \mathcal{G}$ given by $\rho(\varphi)=\lambda M . \sum_{x \in M} \varphi(x)$, where $U$ is the forgetful functor Meas $\rightarrow$ Sets, with the discrete measure functor $D:$ Sets $\rightarrow$ Meas as left adjoint. Using $\rho$ we can transform any Markov chain, i.e. a $\mathcal{D}_{f}$-coalgebra in Sets, $c: X \rightarrow \mathcal{D}_{f}(X)$ into a $U \mathcal{G} D$-coalgebra in Sets, by

$$
\left(X \stackrel{c}{\longrightarrow} \mathcal{D}_{f}(X)=\mathcal{D}_{f} U D(X)\right) \longmapsto\left(X \stackrel{c}{\longrightarrow} \mathcal{D}_{f} U D(X) \stackrel{\rho_{D X}}{\longrightarrow} U \mathcal{G} D(X)\right) .
$$

The latter coalgebras are in one-one correspondence, via the adjunction $D \dashv U$, with $\mathcal{G}$-coalgebras $D(X) \rightarrow \mathcal{G} D(X)$ in Meas with carriers discrete measure spaces.

$$
\begin{gathered}
X \longrightarrow U \mathcal{G} D(X) \quad \text { in Sets } \\
\hline D(X) \longrightarrow \mathcal{G} D(X) \quad \text { in Meas }
\end{gathered}
$$

We use the term discrete Markov process both for a $\mathcal{G}$-coalgebra with carrier discrete measure space in Meas, and for the corresponding $U \mathcal{G} D$-coalgebra in Sets. The whole picture is shown in the following diagram:

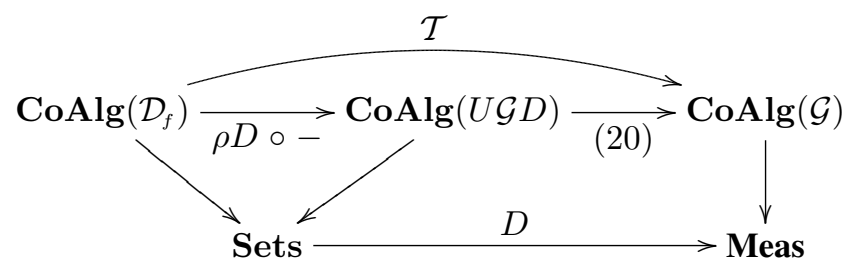

where the square on the right is a pullback of functors.

An important but non-trivial result, see Theorem 18 below, is that behavioural equivalence on a Markov chain $c$ and on the corresponding discrete Markov processes $\mathcal{T}(c)$ coincide. Also the logical theories coincide. This leads to a direct proof of expressivity for Markov chains from expressivity for Markov processes. We can also obtain expressivity for Markov chains from expressivity for Markov processes in an indirect way, using Theorem 4, without explicitly comparing behavioural equivalence. Both proofs are presented below. 


\section{Behavioural equivalence coincides}

The relationship between Markov chains and Markov processes is made explicit with the following theorem. It states that Markov chains can be embedded in the class of Markov processes, namely as discrete Markov processes.

Theorem 18 The translation functor $\left(c: X \rightarrow \mathcal{D}_{f}(X)\right) \mapsto(\mathcal{T}(c): D(X) \rightarrow \mathcal{G} D(X))$ from (21) preserves and reflects behavioural equivalence: for two states $x, x^{\prime} \in X$ we have

$$
x \approx x^{\prime} \text { in } c \Longleftrightarrow x \approx x^{\prime} \text { in } \mathcal{T}(c) .
$$

The proof outlines the main steps and refers to auxiliary results in Appendix A.

Proof. The direction $(\Rightarrow)$ is obvious, by functoriality. The reverse direction is done in two steps. Lemma 24 tells that the functor $\rho D \circ-$ in (21) reflects behavioural equivalence, so it remains to show that the functor labeled with (20) does.

We use that the category Meas is cocomplete. Its colimits are constructed as in Sets, with those subsets measurable that make the coprojections measurable functions, see also [8]. These colimits are inherited by $\operatorname{CoAlg}(\mathcal{G})$. For an arbitrary coalgebra $c: \mathcal{X} \rightarrow \mathcal{G}(\mathcal{X})$ we can consider all abstract epis $h: \mathcal{X} \rightarrow \mathcal{Y}$ with coalgebra $d: \mathcal{Y} \rightarrow \mathcal{G}(\mathcal{Y})$ forming a homomorphism. Since such abstract epis are surjections, they correspond ${ }^{1}$ to equivalence relations on the underlying set of $\mathcal{X}$. Hence these pairs $(h, d)$ form a proper set, and so we can form their joint pushout in:

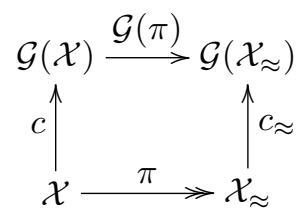

Then $\approx \subseteq \operatorname{ker}(\pi)=\left\{\left(x, x^{\prime}\right) \mid \pi(x)=\pi\left(x^{\prime}\right)\right\}$, by construction.

If we apply this starting from a discrete Markov process $c: D(X) \rightarrow \mathcal{G} D(X)$ we also obtain such a homomorphism $\pi: D(X) \rightarrow \mathcal{Y}$ with $\approx \subseteq \operatorname{ker}(\pi)$. Lemma 25.3 says that $\mathcal{Y}$ then "separates points", and Lemma 27 that $\pi$ is a homomorphism to a discrete $\mathcal{G}$-coalgebra on the carrier of $\mathcal{Y}$. This completes the proof.

\section{Relating the logics and the expressivity results}

We will now relate the logics for Markov chains and Markov processes, and show that the expressivity result for chains follows from the expressivity result for processes. We start by comparing the logic interpretations for Markov chains and Markov processes. In order to disambiguate the two modal operators $L_{r}$ for chains (18) and processes (19) we shall now write them with additional superscripts, namely:

$$
\begin{aligned}
\mathcal{P}(X) \stackrel{L_{r}^{\mathcal{D}_{f}}}{\longrightarrow} \mathcal{P} \mathcal{D}_{f}(X) \quad \text { is } \quad S \longmapsto\left\{\varphi \in \mathcal{D}_{f}(X) \mid \sum_{x \in S} \varphi(x) \geq r\right\} \\
\mathcal{S}(\mathcal{X}) \stackrel{L_{r}^{\mathcal{G}}}{\longrightarrow} \mathcal{S} \mathcal{G}(\mathcal{X}) \quad \text { is } \quad M \longmapsto\{\varphi \in \mathcal{G}(\mathcal{X}) \mid \varphi(M) \geq r\} .
\end{aligned}
$$

\footnotetext{
${ }^{1}$ To be precise, equivalence classes of surjections $X \rightarrow \bullet$ correspond to equivalence relations on $X$.
} 
They give rise to natural transformations:

$$
K \mathcal{P} \stackrel{\otimes^{\mathcal{D}_{f}}}{\longrightarrow} \mathcal{P} \mathcal{D}_{f} \quad \text { and } \quad K \mathcal{S} \stackrel{\otimes^{\mathcal{G}}}{\longrightarrow} \mathcal{S G}
$$

with transposes:

$$
\mathcal{D}_{f} \mathcal{F} \stackrel{\bar{\otimes}^{\mathcal{D}_{f}}}{\longrightarrow} \mathcal{F} K \quad \text { and } \quad \mathcal{G} \mathcal{F} \stackrel{\bar{\nabla}^{\mathcal{G}}}{\longrightarrow} \mathcal{F} K
$$

Lemma 19 For a set $X$, a distribution $\varphi \in \mathcal{D}_{f}(X)$ and a subset $S \in \mathcal{P}(X)=\mathcal{S}(D X)$, where $D(X)$ is the set $X$ with the discrete $\sigma$-algebra $\mathcal{P}(X)$, one has:

$$
\varphi \in L_{r}^{\mathcal{D}_{f}}(S) \Longleftrightarrow \rho(\varphi) \in L_{r}^{\mathcal{G}}(S) .
$$

In a diagram:

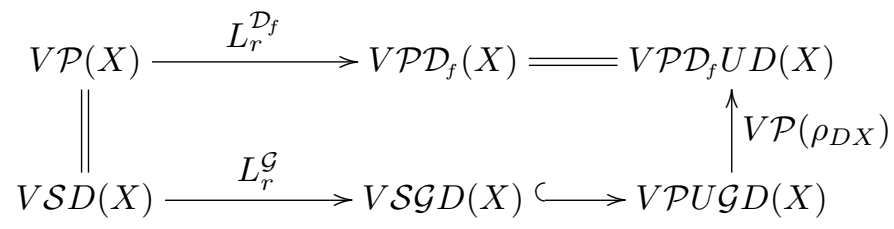

where $V$ is the forgetful functor MSL $\rightarrow$ PoSets. As a result:

$$
\otimes^{\mathcal{D}_{f}}=\mathcal{P}(\rho) \circ \otimes^{\mathcal{G}} \quad \text { and } \quad \bar{\nabla}^{\mathcal{D}_{f}}=U\left(\bar{\nabla}^{\mathcal{G}}\right) \circ \rho \mathcal{F},
$$

where the latter equation involves the diagram:

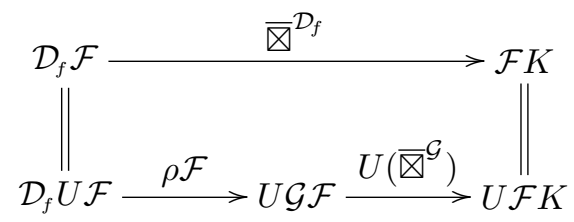

Proof. By unravelling the definitions we get:

$$
\begin{aligned}
\varphi \in L_{r}^{\mathcal{D}_{f}}(S) & \Longleftrightarrow \rho(\varphi)(S)=\sum_{x \in S} \varphi(x) \geq r \\
& \Longleftrightarrow \rho(\varphi) \in L_{r}^{\mathcal{G}}(S) .
\end{aligned}
$$

Then:

$$
\begin{aligned}
\boldsymbol{\otimes}^{\mathcal{D}_{f}} & =\left[\xi \circ H\left(L_{r}^{\mathcal{D}_{f}}\right)\right]_{r} \\
& =\left[\xi \circ H\left(V \mathcal{P}(\rho) \circ L_{r}^{\mathcal{G}}\right)\right]_{r} \\
& =\left[\mathcal{P}(\rho) \circ \xi \circ H\left(L_{r}^{\mathcal{G}}\right)\right]_{r} \\
& =\mathcal{P}(\rho) \circ\left[\xi \circ H\left(L_{r}^{\mathcal{G}}\right)\right]_{r} \\
& =\mathcal{P}(\rho) \circ \nabla^{\mathcal{G}} .
\end{aligned}
$$


Now for $\Phi \in \mathcal{D}_{f} \mathcal{F}(A)$,

$$
\begin{aligned}
\left(U\left(\bar{\nabla}^{\mathcal{G}}\right) \circ \rho_{\mathcal{F}(A)}\right)(\Phi) & =\left\{\psi \in K A \mid \rho(\Phi) \in \nabla^{\mathcal{G}}(K(\eta)(\psi))\right\} \\
& =\left\{\psi \in K A \mid \Phi \in \rho^{-1} \nabla^{\mathcal{G}}(K(\eta)(\psi))\right\} \\
& =\left\{\psi \in K A \mid \Phi \in\left(\mathcal{P}(\rho) \circ \nabla^{\mathcal{G}}(K(\eta)(\psi))\right)\right\} \\
& =\left\{\psi \in K A \mid \Phi \in \nabla^{\mathcal{D}_{f}}(K(\eta)(\psi))\right\} \\
& =\bar{\nabla}^{\mathcal{D}_{f}}(\Phi) .
\end{aligned}
$$

The next result shows that the translation functor does not change the theory maps.

Lemma 20 For a coalgebra $c: X \rightarrow \mathcal{D}_{f}(X)$ let $\llbracket-\rrbracket^{\mathcal{D}_{f}}$ be the corresponding interpretation map from (8) arising from $\nabla^{\mathcal{D}_{f}}$, and let $\llbracket-\rrbracket^{\mathcal{G}}$ correspond to $\mathcal{T}(c): D(X) \rightarrow$ $\mathcal{G}(D(X))$ with $\otimes^{\mathcal{G}}$. Then

$$
\llbracket-\rrbracket^{\mathcal{D}_{f}}=\llbracket-\rrbracket^{\mathcal{G}} \quad \text { and } \quad t h^{\mathcal{D}_{f}}=t h^{\mathcal{G}}
$$

where $t h^{\mathcal{D}_{f}}$ and $t^{\mathcal{G}}$ are the transposes of $\llbracket-\rrbracket^{\mathcal{D}_{f}}$ and $\llbracket-\rrbracket^{\mathcal{G}}$, obtained via the dual adjunctions $\mathcal{F} \dashv \mathcal{P}$ from (4) and $\mathcal{F} \dashv \mathcal{S}$ from (5).

Proof. We obtain $\llbracket-\rrbracket^{\mathcal{D}_{f}}=\llbracket-\rrbracket^{\mathcal{G}}$ by initiality in:

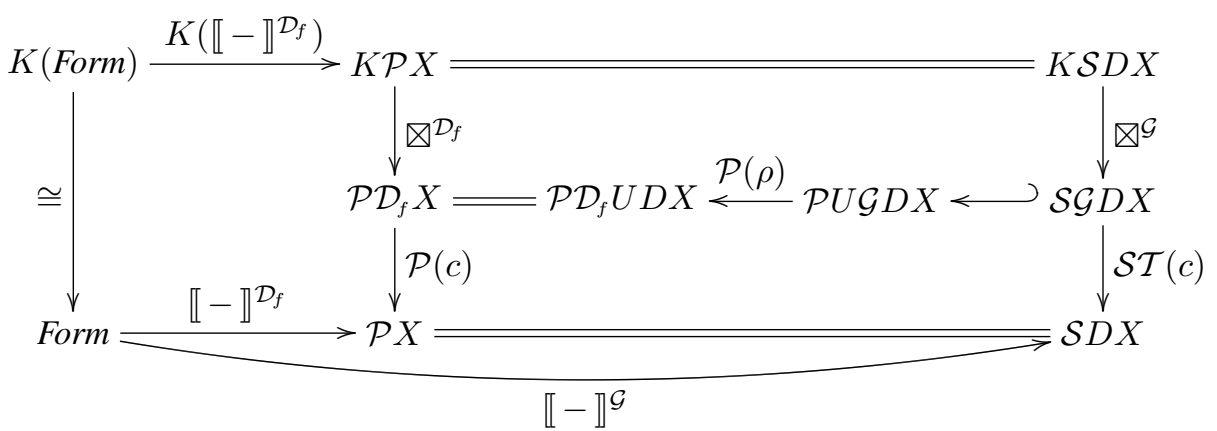

The upper-right square commutes by the previous lemma, and the lower-right one by construction of $\mathcal{T}(c)$.

Hence also $t h^{\mathcal{D}_{f}}=t h^{\mathcal{G}}$, formally as maps $t h^{\mathcal{D}_{f}}: X \rightarrow \mathcal{F}($ Form $)$ in Sets and $t h^{\mathcal{G}}: D X \rightarrow \mathcal{F}($ Form $)$ in Meas.

Corollary 21 Expressivity of modal logic for Markov chains follows from expressivity for Markov processes.

Proof. By the earlier results, using expressivity for Markov processes in $(*)$ :

$$
\begin{aligned}
& x \approx x^{\prime} \text { for } X \stackrel{c}{\longrightarrow} \mathcal{D}_{f}(X) \stackrel{\text { Thm. 18 }}{\Longleftrightarrow} x \approx x^{\prime} \text { for } D(X) \stackrel{\mathcal{T}(c)}{\longrightarrow} \mathcal{G} D(X) \\
& \stackrel{(*)}{\Longleftrightarrow} t h^{\mathcal{G}}(x)=t h^{\mathcal{G}}\left(x^{\prime}\right) \text { for } D(X) \stackrel{\mathcal{T}(c)}{\longrightarrow} \mathcal{G} D(X) \\
& \stackrel{\text { Lem. 20 }}{\Longleftrightarrow} t h^{\mathcal{D}_{f}}(x)=t h^{\mathcal{D}_{f}}\left(x^{\prime}\right) \text { for } X \stackrel{c}{\longrightarrow} \mathcal{D}_{f}(X) .
\end{aligned}
$$


We can also obtain expressivity for Markov chains from expressivity for Markov processes in an indirect way, using Theorem 4, without explicitly comparing behavioural and logical equivalence. It follows from the next property, the proof of which is in Appendix B.

Lemma 22 The natural transformation $\rho \mathcal{F}: \mathcal{D}_{f} U \mathcal{F} \Rightarrow U \mathcal{G F}$ is componentwise mono.

Corollary 23 Expressivity of modal logic for Markov chains follows from expressivity for Markov processes, in the sense that $\bar{\nabla}^{\mathcal{D}_{f}}$ is componentwise mono because $\bar{\nabla}^{\mathcal{G}}$ is.

Proof. By Theorem 17 we know that $\bar{\nabla}^{\mathcal{G}}$ is componentwise mono. Hence so is $U\left(\bar{\nabla}^{\mathcal{G}}\right)$, because the forgetful functor $U$ : Meas $\rightarrow$ Sets is a right adjoint (and thus preserves monos). By Lemma 19 we know that $\bar{\nabla}^{\mathcal{D}_{f}}=U\left(\bar{\nabla}^{\mathcal{G}}\right) \circ \rho \mathcal{F}$. Lemma 22 shows that $\rho \mathcal{F}$ is componentwise mono. This completes the alternative proof.

\section{Conclusions}

We have analysed the semantics and logic of four examples of possibilistic and probabilistic state-based systems in a uniform categorical framework and proved expressivity in each of these cases.

\section{Acknowledgements}

We thank Harald Woracek and the anonymous referee for many helpful suggestions that lead to significant improvements of the paper. We also thank Alexander Kurz and Ernst Erich Doberkat for providing some background information.

\section{References}

1. S. Abramsky. Domain theory in logical form. Ann. Pure \& Appl. Logic, 51(1/2):1-77, 1991.

2. M. Barr and Ch. Wells. Toposes, Triples and Theories. Springer, Berlin, 1985. Revised and corrected version available from URL: www. cwru.edu/artsci/math/wells/pub/ttt.html.

3. F. Bartels, A. Sokolova, and E. de Vink. A hierarchy of probabilistic system types. Theor. Comp. Sci., 327(1-2):3-22, 2004.

4. P. Billingsley. Probability and Measure. Wiley-Interscience, New York, 1995.

5. M. Bonsangue and A. Kurz. Duality for logics of transition systems. In V. Sassone, editor, Foundations of Software Science and Computation Structures, number 3441 in Lect. Notes Comp. Sci., pages 455-469. Springer, Berlin, 2006.

6. M. Bonsangue and A. Kurz. Presenting functors by operations and equations. In L. Aceto and A. Ingólfsdóttir, editors, Foundations of Software Science and Computation Structures, number 3921 in Lect. Notes Comp. Sci., pages 172-186. Springer, Berlin, 2006.

7. C. Cïrstea and D. Pattinson. Modular proof systems for coalgebraic logics. Theor. Comp. Sci., 388:83-108, 2007.

8. V. Danos, J. Desharnais, F. Laviolette, and P. Panangaden. Bisimulation and cocongruence for probabilistic systems. Inf. \& Comp., 204:503-523, 2006. 
9. J. Desharnais, A. Edalat, and P. Panangaden. A logical characterization of bisimulation for labeled markov processes. In Logic in Computer Science, 1998.

10. J. Desharnais, A. Edalat, and P. Panangaden. Bisimulation for labeled Markov processes. Inf. \& Comp., 179(2):163-193, 2002.

11. E.-E. Doberkat. Eilenberg-Moore algebras for stochastic relations. Inf. \& Comp., 204(12):1756-1781, 2006.

12. M. Giry. A categorical approach to probability theory. In B. Banaschewski, editor, Categorical Aspects of Topology and Analysis, number 915 in Lect. Notes Math., pages 68-85. Springer, Berlin, 1982.

13. M. Hennessy and R. Milner. On observing non-determinism and concurrency. In J.W. de Bakker and J. van Leeuwen, editors, Mathematical Foundations of Computer Science, number 85 in Lect. Notes Comp. Sci., pages 299-309. Springer, Berlin, 1980.

14. C. Hermida. Fibrations, Logical Predicates and Indeterminates. PhD thesis, Univ. Edinburgh, 1993. Techn. rep. LFCS-93-277. Also available as Aarhus Univ. DAIMI Techn. rep. PB-462.

15. C. Hermida and B. Jacobs. Structural induction and coinduction in a fibrational setting. Inf. \& Comp., 145:107-152, 1998.

16. B. Jacobs. Many-sorted coalgebraic modal logic: a model-theoretic study. Inf. Théor. et Appl., 35(1):31-59, 2001.

17. B. Jacobs and J. Rutten. A tutorial on (co)algebras and (co)induction. EATCS Bulletin, 62:222-259, 1997.

18. P.T. Johnstone. Stone Spaces. Number 3 in Cambridge Studies in Advanced Mathematics. Cambridge Univ. Press, 1982.

19. B. Klin. Coalgebraic modal logic beyond sets. In M. Fiore, editor, Mathematical Foundations of Programming Semantics, number 173 in Elect. Notes in Theor. Comp. Sci. Elsevier, Amsterdam, 2007.

20. C. Kupke, A. Kurz, and D. Pattinson. Algebraic semantics for coalgebraic logics. In Coalgebraic Methods in Computer Science, number 106 in Elect. Notes in Theor. Comp. Sci., pages 219-241. Elsevier, Amsterdam, 2004.

21. C. Kupke, A. Kurz, and Y. Venema. Stone coalgebras. In H.P. Gumm, editor, Coalgebraic Methods in Computer Science, number 82(1) in Elect. Notes in Theor. Comp. Sci. Elsevier, Amsterdam, 2003.

22. C. Kupke, A. Kurz, and Y. Venema. Stone coalgebras. Theor. Comp. Sci., 327(1-2):109-134, 2004.

23. A. Kurz. Specifying coalgebras with modal logic. Theor. Comp. Sci., 260(1-2):119-138, 2001.

24. A. Kurz and D. Pattinson. Coalgebraic modal logic of finite rank. Math. Struct. in Comp. Sci., 15(3):453-473, 2005.

25. K.G. Larsen and A. Skou. Bisimulation through probabilistic testing. Inf. \& Comp., 94:1-28, 1991.

26. L.S. Moss. Coalgebraic logic. Ann. Pure \& Appl. Logic, 96(1-3):277-317, 1999. Erratum in Ann. Pure \& Appl. Logic, 99(1-3):241-259, 1999.

27. D. Pattinson. Coalgebraic modal logic: Soundness, completeness and decidability of local consequence. Theor. Comp. Sci., 309(1-3):177-193, 2003.

28. D. Pattinson. An introduction to the theory of coalgebras. Course notes at the North American Summer School in Logic, Language and Information (NASSLLI), 2003.

29. D. Pavlović, M. Mislove, and J. Worrell. Testing semantics: Connecting processes and process logics. In M. Johnson and V. Vene, editors, Algebraic Methods and Software Technology, number 4019 in Lect. Notes Comp. Sci., pages 308-322. Springer, Berlin, 2006.

30. J. Rutten. Universal coalgebra: a theory of systems. Theor. Comp. Sci., 249:3-80, 2000. 
31. L. Schröder. Expressivity of coalgebraic modal logic: The limits and beyond. In V. Sassone, editor, Foundations of Software Science and Computation Structures, number 3441 in Lect. Notes Comp. Sci., pages 440-454. Springer, Berlin, 2005.

32. L. Schröder. A finite model construction for coalgebraic modal logic. Journ. of Logic and Algebraic Programming, 73:97-110, 2007.

33. E.P. de Vink and J.J.M.M. Rutten. Bisimulation for probabilistic transition systems: a coalgebraic approach. Theor. Comp. Sci., 221:271-293, 1999.

\section{Appendix}

\section{A Auxiliary results for the proof of Theorem 18}

Lemma 24 The mapping $\left(c: X \rightarrow \mathcal{D}_{f}(X)\right) \mapsto\left(\rho_{D X} \circ c: X \rightarrow U \mathcal{G} D(X)\right)$ from (21) preserves and reflects behavioural equivalence: for two states $x, x^{\prime} \in X$ we have

$$
x \approx x^{\prime} \text { in } c \Longleftrightarrow x \approx x^{\prime} \text { in } X \rightarrow U \mathcal{G} D X .
$$

Proof. Behavioural equivalence preservation is direct by functoriality: if $h(x)=h\left(x^{\prime}\right)$ for a homomorphism $h: X \rightarrow Y$ from $c: X \rightarrow \mathcal{D}_{f}(X)$ to $d: Y \rightarrow \mathcal{D}_{f}(Y)$, then $h$ is also a homomorphism from $\rho_{D X} \circ c$ to $\rho_{D Y} \circ d$, by naturality of $\rho$.

For the reflection, assume $h(x)=h\left(x^{\prime}\right)$ for a coalgebra homomorphism $h$ from $\rho_{D X} \circ c: X \rightarrow U \mathcal{G} D(X)$ to $d: Y \rightarrow Y \mathcal{G} D(Y)$. As noted before, see (7), we may assume that such an $h$ is an abstract epi. Furthermore, the natural transformation $\rho D: \mathcal{D}_{f}=\mathcal{D}_{f} U D \Rightarrow U \mathcal{G} D$ is componentwise mono, resulting in the existence of the dashed arrow $d^{\prime}$ in:

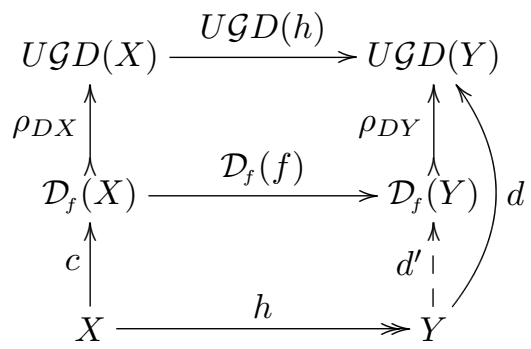

This $d^{\prime}$ is obtained by diagonal fill-in. It proves that $x \approx x^{\prime}$ in $c$.

For an arbitrary function $f$ we write $\operatorname{ker}(f)=\left\{\left(x, x^{\prime}\right) \mid f(x)=f\left(x^{\prime}\right)\right\}$ for the kernel equivalence relation. We recall that a measure space $\mathcal{X}=\left(X, S_{X}\right)$ separates points if for different $x, x^{\prime} \in X$, there exists $M \in S_{X}$ with $x \in M, x^{\prime} \notin M$.

Lemma 25 Let $c: \mathcal{X} \rightarrow \mathcal{G}(\mathcal{X})$ in Meas be a $\mathcal{G}$-coalgebra, with $\mathcal{X}=\left(X, S_{X}\right)$. Then

1. $\mathcal{R}\left(S_{X}\right) \subseteq \operatorname{ker}(c)$, where $\mathcal{R}\left(S_{X}\right)=\left\{\left(x, x^{\prime}\right) \mid \forall M \in S_{X} .\left(x \in M \Leftrightarrow x^{\prime} \in M\right)\right\}$;

2. $\operatorname{ker}(c) \subseteq \approx_{c}$;

3. If $\approx_{c} \subseteq \operatorname{ker}(h)$ for a homomorphism $(\mathcal{X} \stackrel{c}{\rightarrow} \mathcal{G}(\mathcal{X})) \stackrel{h}{\rightarrow}(\mathcal{Y} \stackrel{d}{\rightarrow} \mathcal{G}(\mathcal{Y}))$, then $\mathcal{Y}$ separates points. 
Proof. 1. Assume $\left(x, x^{\prime}\right) \in \mathcal{R}\left(S_{X}\right)$. For each $M \in S_{X}$ and $r \in \mathbb{Q} \cap[0,1]$, one has $L_{r}(M)=\{\varphi \in \mathcal{G}(\mathcal{X}) \mid \varphi(M) \geq r\} \in \mathcal{S G}(\mathcal{X})$ and so $c^{-1}\left(L_{r}(M)\right) \in S_{X}$, so that, by definition of $\mathcal{R}\left(S_{X}\right)$,

$$
x \in c^{-1}\left(L_{r}(M)\right) \Leftrightarrow x^{\prime} \in c^{-1}\left(L_{r}(M)\right) \text { i.e. } \quad c(x)(M) \geq r \Leftrightarrow c\left(x^{\prime}\right)(M) \geq r .
$$

The latter yields $c(x)=c\left(x^{\prime}\right)$, and thus $\left(x, x^{\prime}\right) \in \operatorname{ker}(c)$.

2. The coalgebra map $c: \mathcal{X} \rightarrow \mathcal{G}(\mathcal{X})$, is a homomorphism from $c$ to $\mathcal{G}(c)$ in:

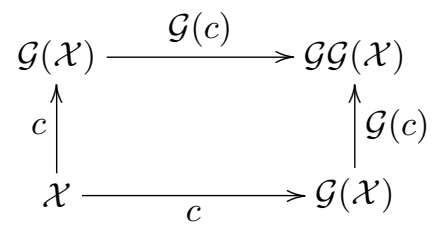

This shows that $\operatorname{ker}(c) \subseteq \approx_{c}$.

3. Assume that $h: X \rightarrow Y$ is a homomorphism from $c$ to $d$. By 1. it is enough to show that $d$ is injective. Hence assume $d(y)=d\left(y^{\prime}\right)$, and write $y=h(x), y^{\prime}=h\left(x^{\prime}\right)$ for certain $x, x^{\prime} \in X$. Then:

$$
\begin{aligned}
\left(x, x^{\prime}\right) & \in(h \times h)^{-1}(\operatorname{ker}(d)) & & \text { by construction } \\
& \subseteq(h \times h)^{-1}\left(\approx_{d}\right) & & \text { by } 2 . \\
& \subseteq \approx_{c} & & \text { because } h \text { is a homomorphism } \\
& \subseteq \operatorname{ker}(h) & & \text { by assumption. }
\end{aligned}
$$

Hence $y=h(x)=h\left(x^{\prime}\right)=y^{\prime}$.

The final step (Lemma 27) uses some basic facts about measure spaces that separate points, which we list first.

Lemma 26 Assume $\mathcal{Y}=\left(Y, S_{Y}\right) \in$ Meas separates points. Then

1. For each countable $U \subseteq Y$ and $y \notin U$ there is an $N \in S_{Y}$ with $U \subseteq N$ and $y \notin N$.

2. For disjoint countable $U, V \subseteq Y$ there is an $N \in S_{Y}$ with $U \subseteq N$ and $V \subseteq \neg N$.

Proof. 1. For each $z \in U$ we have $z \neq y$ so that there is an $N_{z} \in S_{Y}$ with $z \in N_{z}$ and $y \notin N_{z}$. Hence we can take $N=\bigcup_{z} N_{z} \in S_{Y}$.

2. For each $y \in V$ there is, by 1., an $N_{y} \in S_{Y}$ with $U \subseteq N_{y}$ and $y \notin N_{y}$. Now we can take $N=\bigcap_{y} N_{y} \in S_{Y}$.

Lemma 27 Assume a discrete $\mathcal{G}$-coalgebra $c: D(X) \rightarrow \mathcal{G} D(X)$ and a surjective homomorphism $h$ from $c$ to some $\mathcal{G}$-coalgebra $d: \mathcal{Y} \rightarrow \mathcal{G}(\mathcal{Y})$. If $\mathcal{Y}=\left(Y, S_{Y}\right)$ separates points, then there exists a discrete $\mathcal{G}$-coalgebra e: $D(Y) \rightarrow \mathcal{G} D(Y)$ such that $h$ is a homomorphism from c to $e$. 
Proof. We define $e: D(Y) \rightarrow \mathcal{G} D(Y)$ as:

$$
e(y)=c(x) \circ h^{-1}
$$

where $h^{-1}: \mathcal{P}(Y) \rightarrow \mathcal{P}(X), y \in Y$, and $x \in X$ is such that $h(x)=y$. In order to show that $e$ is well-defined, we must show that:

$$
c\left(x_{1}\right) \circ h^{-1}=c\left(x_{2}\right) \circ h^{-1}
$$

whenever $h\left(x_{1}\right)=h\left(x_{2}\right)$. Note that if $e$ is well-defined, then it is certainly measurable and $h$ is a homomorphism from $c$ to $e$.

What we know is that if $h\left(x_{1}\right)=h\left(x_{2}\right)$, then also $(d \circ h)\left(x_{1}\right)=(d \circ h)\left(x_{2}\right)$ and using that $h$ is a homomorphism from $c$ to $d$, we get:

$$
\left(c\left(x_{1}\right) \circ h^{-1}\right)(N)=\left(c\left(x_{2}\right) \circ h^{-1}\right)(N)
$$

for all $N \in S_{Y}$.

So, assume $h\left(x_{1}\right)=h\left(x_{2}\right)$ and $U \subseteq Y$ arbitrary. We will construct a subset $N \in$ $S_{Y}$ such that:

$$
\left(c\left(x_{i}\right) \circ h^{-1}\right)(U)=\left(c\left(x_{i}\right) \circ h^{-1}\right)(N)
$$

for $i \in\{1,2\}$ and this will complete the proof.

The subset $S=\left\{x \in X \mid c\left(x_{1}\right)(\{x\}) \neq 0 \vee c\left(x_{2}\right)(\{x\}) \neq 0\right\}$ is countable. Write:

$$
U_{1}=h\left(S \cap h^{-1}(U)\right), \quad U_{2}=h\left(S \cap h^{-1}(\neg U)\right) .
$$

Clearly, $U_{1}, U_{2} \subseteq Y$ are countable since $S$ is. Moreover, they are disjoint: if $y \in$ $U_{1} \cap U_{2}$, then $y=h\left(x_{1}\right)=h\left(x_{2}\right)$ for some $x_{1} \in h^{-1}(U)$ and $x_{2} \in h^{-1}(\neg U)$, which implies that $y=h\left(x_{1}\right) \in U$ and $y=h\left(x_{2}\right) \in \neg U$, a contradiction. Hence, by Lemma 26, there exists $N \in S_{Y}$ such that $U_{1} \subseteq N$ and $U_{2} \subseteq \neg N$. We are going to show that

$$
S \cap h^{-1}(U)=S \cap h^{-1}(N) .
$$

Indeed, if $x \in S \cap h^{-1}(U)$, then $h(x) \in U_{1} \subseteq N$, so $x \in h^{-1}(N)$ and also $x \in$ $S \cap h^{-1}(N)$. For the reverse inclusion, if $x \in S \cap h^{-1}(N)$, then we either have $x \in$ $h^{-1}(U)$ or $x \in h^{-1}(\neg U)$. But the latter is not possible since it implies that $h(x) \in U_{2}$ so $h(x) \notin N$, a contradiction. Hence $x \in h^{-1}(U)$, and so $x \in S \cap h^{-1}(U)$.

We have shown that $S \cap h^{-1}(U)=S \cap h^{-1}(N)$. Now, for $i \in\{1,2\}$, we have

$$
\begin{aligned}
c\left(x_{i}\right)\left(h^{-1}(U)\right) & =\sum_{x \in S \cap h^{-1}(U)} c\left(x_{i}\right)(\{x\}) \\
& =\sum_{x \in S \cap h^{-1}(N)} c\left(x_{i}\right)(\{x\}) \\
& =c\left(x_{i}\right)\left(h^{-1}(N)\right)
\end{aligned}
$$

which completes the proof. 


\section{B Proof of Lemma 22}

For a meet semilattice $A$ we need to show that the mapping $\rho_{\mathcal{F}(A)}: \mathcal{D}_{f} \mathcal{F}(A) \rightarrow \mathcal{G F}(A)$, given by $\rho(\Phi)=\lambda M \in \mathcal{S F}(A) . \Phi(M)$ is injective-where, as before, $\Phi(M)=$ $\sum_{\alpha \in M} \Phi(\alpha)$. Assume therefore $\Phi, \Psi \in \mathcal{D}_{f} \mathcal{F}(A)$ satisfy $\rho(\Phi)=\rho(\Psi)$. In order to show $\Phi=\Psi$ we assume an arbitrary $\alpha \in \mathcal{F}(A)$ and wish to show $\Phi(\alpha)=\Psi(\alpha)$.

Let $S=\operatorname{supp}(\Phi) \cup \operatorname{supp}(\Psi)$ be the join of the two (finite) supports. We may assume $\alpha \in S$, because otherwise $\Phi(\alpha)=0=\Psi(\alpha)$ and we are done. We form two subsets $B, C \subseteq A$ in the following way. For each $\beta \in S-\{\alpha\}$ we have $\beta \neq \alpha$, so that either $\exists b \in \alpha . b \notin \beta$ or $\exists c \in \beta . c \notin \alpha$. In the first case we choose such a $b \in \alpha-\beta$ and put it in $B$, and in the second case we take a $c \in \beta-\alpha$ and put it in $C$. Since $S$ is finite both $B$ and $C$ are finite (and obtained in finitely many steps). We now define $M \subseteq \mathcal{F}(A)$ as:

$$
\begin{aligned}
M & =\{\gamma \in \mathcal{F}(A) \mid B \subseteq \gamma \text { and } C \cap \gamma=\emptyset\} \\
& =\left(\bigcap_{b \in B} \eta(b)\right) \cap\left(\bigcap_{c \in C} \neg \eta(c)\right) .
\end{aligned}
$$

This second line describes $M$ as finite intersection of measurable subsets. Hence $M \in$ $\mathcal{S F}(A)$ so that $\Phi(M)=\Psi(M)$ since $\rho(\Phi)=\rho(\Psi)$.

Next we claim:

$$
M \cap S=\{\alpha\}
$$

The inclusion $(\supseteq)$ is obvious by construction of $B, C$. For $(\subseteq)$ assume $\gamma \in M \cap S$, but $\gamma \neq \alpha$. Then we have constructed either:

- a $b \in B$ with $b \in \alpha-\gamma$; this is impossible since $B \subseteq \gamma$.

- a $c \in C$ with $c \in \gamma-\alpha$. But since $C \cap \gamma=\emptyset$ this is also impossible.

Hence $\gamma=\alpha$.

We now have $\Phi(\alpha)=\Phi(\{\alpha\})=\Phi(M \cap S)=\Phi(M)=\Psi(M)=\Psi(\alpha)$, as required. Thus $\rho_{\mathcal{F}(A)}$ is injective. 